\title{
La técnica Delphi para la validación de escalas de medida: las variables innovación y reputación dentro de almazaras cooperativas
}

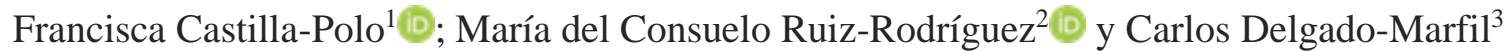

Recibido: 26 de noviembre de 2019 / Aceptado: 26 de mayo de 2020 / Publicado: 4 de noviembre de 2020

\begin{abstract}
Resumen. Nuestro estudio analiza el papel de la innovación en el logro de un mejor posicionamiento estratégico de almazaras cooperativas a través de su reputación. La necesidad de diferenciación en la industria del aceite de oliva se considera una cuestión fundamental en su futuro. Lo anterior justifica nuestro objetivo de diseñar una escala de medida para abordar la medición de la innovación cooperativa al entenderla fundamental para mejorar la reputación de estas empresas y, por extensión, para diferenciarse en un mercado tan complejo como el del aceite de oliva. La metodología utilizada ha incorporado dos fases. La revisión bibliográfica ha sido fundamental para la creación de un modelo conceptual que vincule a estos dos activos: innovación y reputación. Como segunda etapa metodológica, la técnica Delphi con expertos del campo de la innovación y del ámbito cooperativo, nos ha permitido la adaptación de la literatura previa al caso concreto que nos ocupa. Nuestros resultados han validado una escala basada en indicadores mayoritariamente de tipo no financiero para abordar la medición de las variables innovación y reputación permitiendo a través de la misma tanto ayudar a una más adecuada gestión de la innovación por parte de los gerentes de almazaras como aprovechar el máximo beneficio en términos reputacionales.
\end{abstract}

Palabras clave: Innovación; Cooperativas; Reputación; Almazaras; Escala; Técnica Delphi.

Claves Econlit: M14; P13; O13.

[en] The Delphi technique for the validation of measurement scales: the variables innovation and reputation within cooperative oil mills

\begin{abstract}
Our study discusses the role of innovation in improving the strategic positioning of cooperative olive oil mills via reputation. The need for differentiation in the olive oil agri-food industry is considered a fundamental issue in its future. This justifies the need to design a scale to assess the cooperative innovation by understanding it as fundamental to improving the reputation of these companies and, by extension, to differentiate themselves in a market as complex as that of olive oil. The methodology used has incorporated two phases. The literature review has been fundamental for the creation of a conceptual model that links these two assets: innovation and reputation. As a second methodological phase, the Delphi Technique with experts in the field of innovation and cooperativism, has allowed us to adapt the previous literature to our specific case. Our results have validated a scale based on indicators, mostly non-financial, to approach the measurement of innovation and reputation variables, allowing through it both to help a more suitable management of innovation by the managers of olive oil mills and to make the most of it in reputational terms.
\end{abstract}

Keywords: Innovation; Cooperatives; Reputation; Oil mills; Scale; Delphi technique.

Sumario. 1. Introducción. 2. Innovación en el ámbito cooperativo oleícola. 3. La gestión reputacional de almazaras cooperativas. 4. Metodología. 5. Resultados. 6. Conclusiones, limitaciones y futuras líneas de investigación. 7. Referencias bibliográficas.

Cómo citar. Castilla-Polo, F.; Ruiz-Rodríguez, M.C.; Delgado-Marfil, C. (2020) La técnica Delphi para la validación de escalas de medida: las variables innovación y reputación dentro de almazaras cooperativas. REVESCO. Revista de Estudios Cooperativos, vol. 136, e71852. https://dx.doi.org/10.5209/reve.71852.

1 Universidad de Jaén, España.

Dirección de correo electrónico: fpolo@ujaen.es.

2 Universidad de Jaén, España.

Dirección de correo electrónico: mcruiz@ujaen.es.

3 Universidad de Jaén, España.

Dirección de correo electrónico: cdmarfil@ hotmail.com. 


\section{Introducción}

El papel de la innovación como un recurso estratégico ha sido ampliamente evidenciado por la literatura académica. Especialmente, ocupa nuestra atención su consideración como intangible desde hace algunos años por la Teoría de Recursos y Capacidades en sus más conocidos artículos (Barney, 2001; Hall, 1992). Desde estos primeros momentos hasta las líneas de investigación más recientes basadas en métricas e impacto, eco-innovación e innovación abierta como vía de cooperación (Foss y Saebi, 2017; Grimaldi et al. 2017; Subramaniam and Youndt, 2005) se ha producido un importante avance apuntado por BuenecheaElberdin (2017) en su revisión estructurada de este intangible.

Ahora bien, por innovación debemos entender todas las actuaciones que, desde un punto de vista radical o incremental, mejoran la producción, gestión y comercialización de las empresas, tal y como lo entiende el Manual de Oslo de la Organization for Economic Cooperation and Development (OECD) (2005). La innovación no sólo debe aplicarse a la fabricación de nuevos productos o prestación de servicios, sino que también la gestión requiere de nuevos avances tendentes a conseguir una dirección empresarial más eficaz y eficiente a todos sus niveles. Por otro lado, se puede afirmar que hoy en día la innovación influye de manera significativa en la parte comercial donde la preocupación por mejorar la reputación es un objetivo cada vez más interesante. La innovación en marketing y la de tipo social serán claves en este último planteamiento.

Convenimos con Henard y Dancin (2010) que la literatura de innovación se ha centrado mayoritariamente en analizar el impacto tangible, dejando un importante campo de estudio para los efectos intangibles, entre los que dichos autores citan a la reputación como promesa de ventaja competitiva sostenible. Incluso, para De Goey et al. (2019), la innovación en el diseño de nuevos productos debe basarse en sus aspectos inmateriales de cara a garantizar la creación de valor. Por lo comentado, nos dedicaremos en este trabajo a cubrir este gap de investigación al respecto de los efectos intangibles derivados de innovar, especialmente, su efecto sobre la creación/mejora de la reputación corporativa. Para Chun (2006), la innovación mejora la reputación si ésta es sostenible, esto es, si está basada en la integridad y en el coraje. Por su parte, Padgett and Moura-Leitte (2012) se ocupan de la relación entre intensidad de la innovación y reputación, corroborando su sentido positivo a la vez que el papel moderador de la responsabilidad social. Höflinger et al. (2018) plantean la influencia de la innovación tecnológica sobre la reputación concluyendo sobre la apreciación que los consumidores realizan del esfuerzo por innovar de las empresas. Otro estudio relevante es realizado por González-Ramos et al. (2018) quienes afirman que las empresas más proactivas a la innovación presentan un mayor compromiso responsable y que éste a su vez se traduce en una mejora de su resultado financiero.

Con estos antecedentes, nuestro objetivo de investigación ha consistido en analizar la innovación en sus diferentes dimensiones como herramienta para la gestión reputacional dentro de la industria productora de aceite de oliva o almazarera aglutinada mayoritariamente bajo la forma jurídica cooperativa ${ }^{4}$. Concretamente, se ha diseñado y validado empíricamente a través de la técnica Delphi un modelo conceptual que definirá cómo medir ambas variables a través de una batería de indicadores mayoritariamente de tipo no financiero.

La justificación de la población elegida se justifica a través del siguiente razonamiento. Se trata de unas sociedades donde la diferenciación resulta vital, tanto por la naturaleza de su producto, el aceite de oliva, que se integra en la problemática de la diferenciación dentro de la agroindustria (Castilla-Polo et al. 2018; Cabrera et al. 2015) como por el elevado grado de homogeneización de infraestructura de estas empresas ya evidenciado desde hace tiempo por informes previos, entre ellos el elaborado por el Ministerio de Agricultura Pesca y Alimentación (MAPA) (2004). Por otro lado, también nos encontramos ante unas sociedades donde la apuesta por sus valores intangibles es igualmente oportuna e imprescindible (CastillaPolo y Ruiz-Rodríguez, 2018) y donde el carácter cooperativo, prioritario dentro de esta industria, matiza las investigaciones realizadas por trabajos previos. Analizar si la innovación puede repercutir en conseguir una mejor reputación permitiría dotarlas de una herramienta para indirectamente conseguir una diferenciación en el mercado y sería un factor clave para favorecer la visibilidad organizacional que desea esta industria.

El diseño metodológico ha incorporado dos fases. En primer lugar, la revisión teórica de las principales bases de datos y publicaciones especializadas tanto en el campo de la innovación y de la reputación como en el del cooperativismo. Ello nos ha permitido concluir sobre el papel de la innovación a través de sus diferentes formas y sobre su incidencia en la reputación, así como sobre aquellos indicadores más utilizados por la literatura en la medición de ambas variables que han constituido el punto de partida para la aplicación de la técnica Delphi en una subsiguiente fase. Se ha contado con la participación de un grupo de expertos en la materia tanto académicos como profesionales que han permitido finalmente validar los indicadores más

4 En torno al $56 \%$ de estas empresas revisten la forma jurídica cooperativa, lo que justifica su relevancia. Disponible en: http://www.esenciadeolivo.es/aceite-de-oliva/produccion/industrias-del-aceite-en-espana/ (acceso octubre 2019). 
apropiados para abordar la medición de ambas variables. La técnica Delphi ha sido empleada en trabajos previos para la validación de escalas, tal es el caso del emprendimiento y de sus factores de éxito por Martínez-García et al. (2019), para el desarrollo de la investigación en el sector agropecuario por Fernández y López (2013) así como en el caso del propio sector del aceite de oliva para estudiar las prioridades en I+D+i por Sanz et al. (2011).

El trabajo se estructura en seis apartados. En los dos siguientes revisaremos el estado de la investigación sobre innovación y reputación dentro de la realidad almazarera y cooperativa. A continuación, la descripción de la metodología utilizada ocupará el cuarto bloque que dará paso al estudio de los principales resultados obtenidos en la quinta sección. Finalizaremos comentando las principales conclusiones alcanzadas, las futuras líneas de trabajo, así como las limitaciones detectadas.

\section{Innovación en el ámbito cooperativo oleícola}

La necesidad de mejorar la competitividad del sector oleícola ha sido revisada ampliamente y desde una perspectiva global por Parras y Gómez-Limón (2017: 16) identificando la necesidad de actuar bajo cuatro amplias líneas, entre las que se incluye una dedicada a "iv) Implementar la transformación digital, la bioeconomía y los demás procesos de innovación". También, la Junta de Andalucía a través de Extenda (2017: 9) viene a delimitar los siguientes ejes estratégicos: la promoción, la eficiencia operativa, la internacionalización y la innovación. Sobre este último eje, recogemos su recomendación específica: "la innovación, sensu lato, no sólo técnica, también comercial, operativa,... esto debería conllevar una modificación de las variables de la competencia actual, fuertemente articulada sobre la variable "precio" de un producto que es mayormente considerado una commodity alimentaria, y debería permitir una mejor posición de las marcas de fabricantes, ahora bajo la presión fuerte de las marcas propias de la distribución". Como se puede observar, la innovación aparece en el futuro del sector entendida en un sentido amplio tal y como recoge De Toro (2017: 426) "esta apuesta por la innovación se ha materializado, no solo en la mejora de los procesos productivos, sino en la innovación aplicada en otras áreas como la gestión, la comunicación, la orientación al cliente, así como en la profesionalización del sector".

Según propone Calabria (2018) debemos diferenciar las estrategias individuales de cada almazara frente a estrategias del sector que pudieran ser abordadas de forma conjunta y a través de actuaciones que contasen con el apoyo político y público necesario con lo que se mitigarían ambos problemas. Partiendo de este último estudio, puede observarse la referencia explícita a la diferenciación a la vez que el papel de la innovación como base de un importante número de las estrategias propuestas para ambas vertientes. Véase Tabla 1.

Tabla. 1. Estrategias de diferenciación

\begin{tabular}{|c|c|c|c|}
\hline \multicolumn{2}{|c|}{ Estrategias conjuntas } & \multicolumn{2}{|c|}{ Estrategias individuales } \\
\hline Fase industrial & Fase comercial & Fase industrial & Fase comercial \\
\hline $\begin{array}{l}\text { Innovación e } \\
\text { investigación aplicada }\end{array}$ & $\begin{array}{l}\text { Participación en } \\
\text { ferias sectoriales }\end{array}$ & $\begin{array}{l}\text { Diferenciación por } \\
\text { enfoque }\end{array}$ & Premios a la calidad \\
\hline & Oleoturismo & $\begin{array}{l}\text { Aceites balsámicos a } \\
\text { los ahumados }\end{array}$ & Endorsement (influencers) \\
\hline & & Envasado y etiquetado & \\
\hline
\end{tabular}

Fuente: Elaboración propia a partir de Calabria (2018)

Tomando en consideración la Tabla 1, nos dedicaremos al estudio de la innovación de tipo individual en estas empresas agrupada bajo dos dimensiones: la innovación en la fase industrial y en la fase comercial. Así, las innovaciones de producto y de tipo organizativo propuestas por el Manual de Oslo (OECD, 2005) se vincularían a la fase industrial que Calabria (2018) propone, mientras que las dos últimas: social y de marketing, parecen asociarse con la fase comercializadora del aceite de oliva ${ }^{5}$. Pasamos a continuación a detallar ambas dimensiones y las proposiciones teóricas derivadas de nuestro estudio.

Debemos aclarar que la innovación social por su amplitud podría haber sido incluida en ambas dimensiones, si bien, parece observarse en la investigación académica que este tipo de innovación suele ser revelada, lo que justificaría su adscripción al bloque comercial. 


\subsection{Innovación en fase industrial}

Innovación en producto. Aunque el modelo de negocio de almazaras contempla un producto fundamental, el aceite de oliva en sus diferentes categorías, también el desarrollo de nuevos productos es una meta a tener en cuenta en el futuro de estas empresas. Siguiendo a Calabria (2018) desde los aceites balsámicos a los ahumados, existe una amplia posibilidad de innovación por parte de almazaras. En los últimos años, debemos destacar el papel del oleoturismo como oferta de cultura y ocio, siendo otra vía para la diversificación de la cartera de almazaras (Carrillo et al. 2019).

Innovación en procesos. Las posibilidades de innovación en procesos dentro de almazaras han sido tratadas de manera más intensiva por la investigación que las relativas al producto. Dentro de las prioridades para España del Instituto Nacional de Investigaciones Agronómicas (INIA) (2014-2017) la eco-innovación y el eco-diseño han resultado prioridades fundamentales (García et al. 2018). Otros estudios se centran en el ámbito productivo, Chiavaro (2014) plantea mejorar el rendimiento del proceso de extracción de la aceituna y Vilar et al. $(2010,2011)$ se refieren a la gestión interna de costes y al rendimiento como cuestión imprescindible en un horizonte a corto/medio plazo. También Peraza et al. (2016) y Caravaca et al. (2006) concluyen que cualquier tipo de certificación en calidad, medioambiental o geográfica resulta ilustrativa de la intención de innovar. Aponte et al. (2015) van más allá indicando que las certificaciones de calidad son un factor clave, reflejo de la capacidad de innovar, mientras que para Bel y Ausín (2007) las certificaciones clave son las de tipo ambiental. Respecto a estas últimas, Sajardo-Moreno y Chaves-Sajardo (2017) concluyen que poseer este tipo de certificación es uno de los elementos diferenciadores en las empresas de Economía Social. También las disfunciones en el gobierno de almazaras cooperativas y la falta de profesionalización en la gestión apuntadas por Ruiz (2006) abren un camino importante para la innovación en procesos organizativos.

\subsection{Innovación en fase comercial}

Innovación en marketing. Consideramos especialmente relevante su relación con la mejora/creación de reputación en cualquier empresa, incluidas almazaras (López-Quesada, 2017; De Sá, 2012; Chun, 2006). Para Montegut et al. (2007) la calidad debe ser la base de la innovación en marketing en cooperativas almazareras por sus efectos sobre el poder de negociación frente a terceros y la subsiguiente mejora reputacional. También resulta crucial el diseño de una web, que capte la atención y que facilite la operación de venta. En este sentido, las webs corporativas y su diseño como vía mejorar la eficiencia económica de almazaras han sido recientemente estudiadas por Bernal et al. (2018).

Innovación social. Representa una excelente oportunidad "para consolidar una imagen y prestigio basados en la diferenciación de sus servicios al reforzar sus acciones sociales" (Sanchís y Campos, 2008: 187). Por sus propios valores y principios, las cooperativas la han trabajado desde sus orígenes, aunque deberían seguir reforzando este papel de cara a la adquisición de diferentes ventajas competitivas (FAECTA, 2016; Hechevarría y Cruz, 2016). Lajara-Camirelli y Server-Izquierdo (2017) plantean que, junto a la propia identidad cooperativa, la innovación social es vital para la competitividad de las cooperativas agroalimentarias. Amo (2018) se refiere específicamente a que ya se está poniendo en práctica la preocupación por innovar en sostenibilidad por parte de las empresas productoras de aceite de oliva. Si bien, para Godino (2018) éstas deberían presentar especial atención a la gestión ambiental que incluye temas tan actuales como la reducción del consumo energético, la sostenibilidad energética y el reciclaje.

\subsection{Indicadores seleccionados para la variable innovación}

Derivada de la condición intangible de la innovación se pueden deducir dos especiales atributos que deben ser tenidos en cuenta a la hora de abordar su medición. En primer lugar, su carácter multidimensional o consideración como constructo integrado por diferentes dimensiones. En nuestro caso concreto, las peculiaridades de la industria oleícola y de las empresas productoras de aceite de oliva nos ha llevado a su agrupación en dos dimensiones: innovaciones para la fase industrial -que abarcarían las innovaciones de producto y de proceso- e innovaciones para la fase comercial -que integraría a las de marketing y de tipo social-. En segundo lugar, apostamos por la necesidad de valoración no financiera a través de indicadores al ser ésta la técnica más utilizada a la hora de proceder a la medición de intangibles (Grasenick y Low, 2004).

Teniendo en cuentas ambas consideraciones, en la Tabla 2 se muestran aquellos indicadores que se han considerado relevantes tras el análisis de la literatura previa en innovación y, concretamente, dentro del 
sector del aceite de oliva. Se han seleccionado 17 indicadores que han cumplido dos requisitos: relevancia para medir la innovación y aplicabilidad al caso concreto de las almazaras cooperativas.

Tabla. 2. Propuesta de indicadores para la variable innovación en almazaras

\begin{tabular}{|c|c|c|c|}
\hline & Indicador propuesto & Justificación & Background \\
\hline \multirow{10}{*}{ 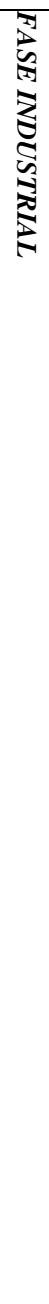 } & $\begin{array}{l}\text { Incorporación de nuevos } \\
\text { productos }\end{array}$ & $\begin{array}{l}\text { La búsqueda de aceites de oliva balsámicos o ahumados, } \\
\text { oleoturismo, entre otros productos innovadores a modo de } \\
\text { ejemplos. }\end{array}$ & $\begin{array}{l}\text { COTEC (2017), EOI (2007), Fernández y } \\
\text { Peña (2009), Lugones } \quad \text { (2009), Sancho } \\
\begin{array}{ll}(2002) & \end{array}\end{array}$ \\
\hline & $\begin{array}{l}\text { Modificación, mejora de } \\
\text { productos existentes }\end{array}$ & $\begin{array}{l}\text { La importancia de conseguir un aceite de oliva de más calidad } \\
\text { ha sido una nota repetida que nos ha llevado a seleccionar este } \\
\text { indicador. }\end{array}$ & $\begin{array}{l}\text { COTEC (2017), Fernández y Peña (2009), } \\
\text { Lugones (2009), Quesada (2008), EOI } \\
\text { (2007), Sancho }(2007,2002)\end{array}$ \\
\hline & $\begin{array}{l}\text { Incorporación de nuevos } \\
\text { procesos }\end{array}$ & $\begin{array}{l}\text { La implantación de nuevos procesos en cualquier actividad de } \\
\text { la almazara, tendentes a conseguir resultados óptimos en el } \\
\text { ámbito de la gestión, producción y organización, así como } \\
\text { concretamente a mejorar la eficiencia y la eficacia en estas } \\
\text { empresas. }\end{array}$ & $\begin{array}{l}\text { COTEC (2017), Fernández y Peña (2009), } \\
\text { Lugones (2009) }\end{array}$ \\
\hline & $\begin{array}{l}\text { Modificación, mejora de } \\
\text { procesos productivos }\end{array}$ & $\begin{array}{l}\text { La renovación de procesos tanto productivos como de gestión } \\
\text { en un sentido amplio también se ha planteado como } \\
\text { imprescindible en este sector. }\end{array}$ & $\begin{array}{l}\text { COTEC (2017), Fernández y Peña (2009), } \\
\text { Lugones (2009), EOI (2007) }\end{array}$ \\
\hline & Certificaciones de calidad & $\begin{array}{l}\text { La importancia de la calidad y de su acreditación ante las } \\
\text { exigencias del consumidor nos lleva a revisar los diferentes } \\
\text { sistemas de certificación. La elección del consumidor puede } \\
\text { estar basada en la posesión de certificaciones de este tipo. }\end{array}$ & Aponte (2015), Cara \\
\hline & $\begin{array}{l}\text { Certificaciones } \\
\text { ambientales }\end{array}$ & $\begin{array}{l}\text { La problemática de los residuos generados por la almazara nos } \\
\text { lleva a identificar el valor de las diferentes certificaciones } \\
\text { ambientales para reducir su impacto en el medio ambiente. }\end{array}$ & $\begin{array}{l}\text { Sajardo-Moreno y Chaves-Sajardo (2017), } \\
\text { Bel y Ausín (2007), Caravaca et al. (2006) }\end{array}$ \\
\hline & $\begin{array}{l}\text { Certificaciones } \\
\text { geográficas }\end{array}$ & $\begin{array}{l}\text { Las denominaciones de origen y sus implicaciones en el aceite } \\
\text { de oliva denotan la importancia del origen y garantizan al } \\
\text { consumidor un nivel de calidad y características específicas } \\
\text { para el producto. }\end{array}$ & $\begin{array}{l}\text { Montegut et al. (2007), Caravaca et al. } \\
\text { (2006) }\end{array}$ \\
\hline & Patentes & $\begin{array}{l}\text { Número de patentes de la almazara como indicador de } \\
\text { innovación y de propiedad de la misma. }\end{array}$ & $\begin{array}{l}\text { COTEC (2017), Aponte (2015), Navarro et } \\
\text { al. (2011), Anlló et al. (2009), González y } \\
\text { Romeu (2009), Perea (2009), Quesada } \\
\text { (2008), EOI (2007), Sancho (2007), } \\
\text { Caravaca et al. (2006) }\end{array}$ \\
\hline & $\begin{array}{l}\text { Inversión en } \\
\text { infraestructuras } \\
\text { innovadoras/tecnológicas }\end{array}$ & $\begin{array}{l}\text { Importe destinado a dotar a la almazara de los medios } \\
\text { estructurales necesarios para conseguir diferentes objetivos, } \\
\text { bien de tipo productivo, comercial u organizativo y poder } \\
\text { alcanzar una posición competitiva de liderazgo en el mercado. }\end{array}$ & $\begin{array}{l}\text { COTEC (2017), Acevedo et al. (2016), } \\
\text { Aponte (2015), Navarro et al. (2011), Anlló } \\
\text { et al. (2009), Fernández y Peña (2009), } \\
\text { Quesada (2008), Caravaca et al. (2006) }\end{array}$ \\
\hline & $\begin{array}{l}\text { Colaboraciones con } \\
\text { universidad, centros de } \\
\text { investigación y otras } \\
\text { instituciones }\end{array}$ & $\begin{array}{l}\text { La participación en proyectos de investigación, centros } \\
\text { tecnológicos y el establecimiento de alianzas es entendida } \\
\text { como un valor clave en el desarrollo de la innovación de } \\
\text { cooperativas almazareras, que les permitirá competir para } \\
\text { conseguir un beneficio mutuo y unir fuerzas para superar } \\
\text { barreras o acometer importantes inversiones. }\end{array}$ & $\begin{array}{l}\text { Escobar et al. (2018), COTEC (2017), } \\
\text { Aponte (2015), Ministerio de Economía y } \\
\text { Competitividad (2013), Navarro et al. } \\
\text { (2011), Anlló et al. (2009), Quesada (2008), } \\
\text { EOI (2007) }\end{array}$ \\
\hline \multirow{7}{*}{ 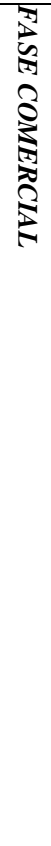 } & $\begin{array}{l}\text { Sistemas de gestión de } \\
\text { clientes }\end{array}$ & $\begin{array}{l}\text { El cliente como grupo de interés clave nos lleva a buscar } \\
\text { herramientas para gestionar de una forma más efectiva las } \\
\text { relaciones con ellos e intentar satisfacer sus necesidades y } \\
\text { expectativas respecto a la almazara. }\end{array}$ & $\begin{array}{l}\text { Fidel et al. (2018), Wang y Xu (2018), } \\
\text { Valmohammadi (2017), Ministerio de } \\
\text { Economía y Competitividad (2013) }\end{array}$ \\
\hline & $\begin{array}{l}\text { Número de marcas } \\
\text { registradas }\end{array}$ & $\begin{array}{l}\text { Como indicador de la capacidad de innovación comercial, de } \\
\text { defensa de su propiedad y de factor de diferenciación para los } \\
\text { clientes. }\end{array}$ & Perea (2009), Quesada (2008), EOI (2007) \\
\hline & $\begin{array}{l}\text { Liderazgo en ventas } \\
\text { envasadas }\end{array}$ & $\begin{array}{l}\text { El intentar acaparar un mayor nivel de aceite envasado dentro } \\
\text { de las ventas cooperativas es una cuestión repetida para } \\
\text { mejorar la situación de estas empresas. }\end{array}$ & Moradi (2017), Gutiérrez y Palacios (2015) \\
\hline & $\begin{array}{l}\text { Presencia ámbito comercio } \\
\text { exterior }\end{array}$ & $\begin{array}{l}\text { La necesidad de búsqueda de mercados donde comercializar el } \\
\text { aceite de oliva nos lleva a buscar fórmulas para conseguir la } \\
\text { internacionalización de estas empresas. }\end{array}$ & $\begin{array}{l}\text { Bretos et al. (2018a), Navarro et al. (2011), } \\
\text { Sancho (2002) }\end{array}$ \\
\hline & $\begin{array}{l}\text { Modificación sistema de } \\
\text { intercambio de } \\
\text { información/TICs }\end{array}$ & $\begin{array}{l}\text { La presencia de sistemas de comunicación internos para el } \\
\text { trasvase de información es fundamental, ayuda a mantener el } \\
\text { flujo comunicativo y se relaciona con la integración de las } \\
\text { nuevas tecnologías en estas empresas, tan demandada por los } \\
\text { autores de este campo. }\end{array}$ & $\begin{array}{l}\text { Bretos et al. (2018b), Fernández et al. } \\
\text { (2015), Jafar y Eshghi (2011), Caballer y } \\
\text { Mocaleano (2004) }\end{array}$ \\
\hline & $\begin{array}{l}\text { Responsabilidad Social } \\
\text { asumida y ejercida }\end{array}$ & $\begin{array}{l}\text { La sostenibilidad de la almazara es fundamental al igual que } \\
\text { para cualquier otro tipo de empresa. Apostar por actuaciones } \\
\text { responsables con el entorno, empleados y medioambiente es } \\
\text { fundamental al igual que en cualquier otra empresa. }\end{array}$ & $\begin{array}{l}\text { Etxezarreta et al. (2018), Fajardo (2018), } \\
\text { Marcuello y Nachar (2013), Benito y } \\
\text { Esteban (2012), García et al. (2011), Mozas } \\
\text { y Puentes (2010) }\end{array}$ \\
\hline & $\begin{array}{l}\text { Visibilidad on line } \\
\text { (número de visitas) }\end{array}$ & $\begin{array}{l}\text { Las importantes exigencias del comercio en internet y la } \\
\text { comercialización a través de este canal han llevado al sector a } \\
\text { preocuparse por su visibilidad on line. }\end{array}$ & $\begin{array}{l}\text { Bernal et al. (2019), Jorge et al. (2019) } \\
\text { Fernández et al. (2016), Fernández et al. } \\
\text { (2015), Moral et al. (2014), EOI (2007), } \\
\text { Meroño y Arcas (2006) }\end{array}$ \\
\hline
\end{tabular}

Fuente: Elaboración propia 
Dentro de la fase industrial destacamos 10 indicadores. En primer lugar, la incorporación de nuevos productos o procesos, o la mejora de los ya existentes, debido a que consideramos relevante la mejora día a día en cualquier empresa, incluidas las que nos ocupan. Las certificaciones de calidad, ambientales y geográficas, características del sector del aceite de oliva, son otros de los indicadores elegidos ya que en este proceso de innovación la almazara debería intentar garantizar a sus principales grupos de interés aspectos tan importantes como la calidad, el origen y su actuación ambiental. Otros indicadores de innovación de la fase industrial seleccionados tienen que ver con la posesión de patentes, la inversión en infraestructuras tecnológicas y la colaboración con universidades u otros centros de investigación con los cuales la almazara intentará definir su posición en el mercado, aumentar su valor en el sector y, en última instancia, obtener ventajas competitivas.

Por otro lado, dentro de la dimensión comercial hemos considerado relevantes 7 indicadores relacionados con el marketing y con objetivos de tipo social. Así, destacamos la importancia de poseer un adecuado sistema de gestión de clientes con el que satisfacer sus necesidades, el número de marcas registradas o tener una posición de liderazgo en el sector. Además, la presencia a nivel internacional debería estar dentro de los objetivos de estas empresas para lo cual será fundamental tanto el comercio vía internet y disponer de un sistema de comunicación on line, como adecuar los sistemas de intercambio de información de la almazara a las nuevas tecnologías. Finalmente, destacamos el papel de la responsabilidad social como garantía de un adecuado comportamiento que las legitime y, máxime dada su condición como entidades de la Economía Social.

\section{La gestión reputacional de almazaras cooperativas}

Según venimos argumentando, nos adentramos en una importante línea de estudio que corrobora el indudable valor de una empresa innovadora dada su relación con la mejora de su visibilidad organizacional (Zhang, 2018; Courtright y Smudde, 2009). Según Lee y Roh (2012), la reputación desempeña un papel fundamental en el desarrollo del interés externo hacia la empresa. Una buena reputación incrementa la confianza de clientes en los productos y servicios ofrecidos actuando como una demanda publicitaria en la decisión de compra (Fombrun y van Riel, 1998). Además, según Corporate Excellence (2019) la reputación participa en la creación de valor entre los stakeholders donde se incluyen los clientes, los proveedores, los empleados, etc. siendo todas estas percepciones las que generan a su vez este intangible. Otros autores plantean que las empresas con elevada reputación gozan de un valor de mercado superior y de una reducción de sus costes de capital (Wang et al. 2016; Cao et al. 2015; Carter y Ruefli, 2006; Roberts y Dowling, 2002; Fombrun, 1996). También los empleados desean trabajar en empresas con excelente reputación y para éstas es más fácil mantener a sus empleados (Bergh et al. 2010; Boyd et al. 2010; Roberts y Dowling, 2002).

La literatura insiste en que no hay una forma más directa de distinguir a una empresa que hacerlo a través de su reputación, puesto que uno de sus principales beneficios es la creación de barreras de acceso al mercado en el sentido planteado por Porter (1979). Si bien, existen escasas evidencias previas que analicen esta variable de forma empírica en el caso agroalimentario y, específicamente dentro del cooperativo. Como un antecedente directo a nuestra investigación, Castilla-Polo et al. (2018) consideran la reputación cooperativa como un constructo integrado por cuatro variables: innovación, responsabilidad social, premios y certificaciones de calidad; Casimiro y Matos (2015) presentan un modelo dotado de antecedentes y de impactos testado en tres cooperativas; y Martínez-Carrasco y Eid (2017) comprueban la imagen positiva de cooperativas frente a sociedades capitalistas con relación a sus beneficios sociales. Se trata de una variable poco estudiada hasta la fecha y que justifica cualquier intento por acotar y definir tanto sus antecedentes como su propia naturaleza.

\subsection{Indicadores seleccionados para la variable reputación}

Al igual que la innovación, la reputación resulta difícil de valorar dada su condición de intangible. Walsh y Beatty (2007) se refieren a su carácter multidimensional, afirmando que su valor depende de diferentes atributos y de diferentes grupos (Corporate Excellence, 2019). De hecho, es precisamente este carácter el que justifica la importancia de los rankings de reputación, valoraciones multidimensionales, multi-stakeholders y dinámicas (generalmente cada año natural). Dentro de ellos, debemos destacar el ranking Fortune, a nivel internacional, que desde sus inicios sigue siendo el más empleado, el ranking elaborado por Reputation Institute, the Global RepTrak® 100, así como el creciente auge para el ámbito español y latinoamericano del ranking MERCO, quien ocupa un papel análogo en nuestro contexto.

Dentro del ámbito cooperativo se ha optado por la consideración de la reputación como constructo integrado por un total de 11 indicadores. Si bien es cierto que existe un ranking para las empresas más 
relevantes a nivel de Economía Social elaborado por $\mathrm{CEPES}^{6}$, no es asimilable al estudio de empresas cooperativas con mejor reputación. Es por este motivo por el que se ha decidido crear una escala propia para esta variable que se ha partido de aquellas empleadas por el ranking MERCO (2018), realizando una adaptación a las peculiaridades de estas empresas. Igualmente, se han añadido variables procedentes de la revisión bibliográfica realizada que entendíamos de aplicación a nuestro caso concreto. En la Tabla 3 podemos encontrar los indicadores seleccionados tras la adaptación de estos indicadores considerados como relevantes por la literatura a las peculiaridades de la industria almazarera.

Tabla. 3. Propuesta de indicadores para la variable reputación en almazaras

\begin{tabular}{|c|c|c|}
\hline Indicador & Justificación & Background \\
\hline $\begin{array}{l}\text { Proyección de } \\
\text { beneficios a corto } \\
\text { y medio plazo }\end{array}$ & $\begin{array}{l}\text { Conocer la rentabilidad se considera un } \\
\text { indicador clave sobre el funcionamiento } \\
\text { empresarial en el corto y medio plazo. } \\
\text { Reputación financiera. }\end{array}$ & $\begin{array}{l}\text { Maestre et al. (2019), MERCO (2018), Ponzi et al. } \\
\text { (2011), Walsh y Beatty (2007), López e Iglesias (2006), } \\
\text { Villafañe (2004), Cravens et al. (2003), Roberts y } \\
\text { Dowling (2002), Fombrun et al. (2000), Fombrum } \\
\text { (1996), Weigelt y Camerer (1988) }\end{array}$ \\
\hline $\begin{array}{l}\text { Calidad de los } \\
\text { productos } \\
\text { ofrecidos }\end{array}$ & $\begin{array}{l}\text { Garantizar un producto de calidad, en este caso, } \\
\text { un aceite de oliva, resulta fundamental para } \\
\text { garantizar la reputación de cualquier almazara. } \\
\text { Reputación de producto. }\end{array}$ & $\begin{array}{l}\text { Schwaiger et al. (2019), Dias (2018), MERCO (2018), } \\
\text { WMAC (2019)7, Olmedo y Martínez (2011), Walsh y } \\
\text { Beatty (2007), Martín et al. (2006), López e Iglesias } \\
\text { (2006), Helm (2005, 2007), Dowling (2004), } \\
\text { Schwaiger (2004), Cravens et al. (2003), De Quevedo } \\
\text { (2003), Wessels (2003), Caruana y Chircop (2000), } \\
\text { Fombrun et al. (2000), Weigelt y Camerer (1988) }\end{array}$ \\
\hline $\begin{array}{l}\text { Acierto estrategia } \\
\text { de la empresa }\end{array}$ & $\begin{array}{l}\text { Definir una estrategia adecuada en la } \\
\text { cooperativa resulta imprescindible para dar a } \\
\text { conocer su visión de futuro al exterior. }\end{array}$ & $\begin{array}{l}\text { Maestre et al. (2019), MERCO (2018), Walsh y Beatty } \\
\text { (2007), López e Iglesias (2006), Helm (2005), Dowling } \\
\text { (2004), Cravens et al. (2003), Fombrun y Shanley } \\
\text { (1990) }\end{array}$ \\
\hline Calidad laboral & $\begin{array}{l}\text { La calidad laboral vendrá definida, en parte, por } \\
\text { el grado de satisfacción de los empleados y la } \\
\text { ausencia de posibles conflictos/litigios. }\end{array}$ & $\begin{array}{l}\text { MERCO (2018), Walsh y Beatty (2007), Helm (2005), } \\
\text { Schwaiger (2004), Wessels (2003), Villafañe (2004), } \\
\text { Cravens et al. (2003), Caruaana y Chircon (2000), } \\
\text { Fombrun et al. (2000), Weigelt y Camerer (1988) }\end{array}$ \\
\hline $\begin{array}{l}\text { Igualdad de } \\
\text { oportunidades }\end{array}$ & $\begin{array}{l}\text { Garantizar la igualdad de género y la existencia } \\
\text { de oportunidades laborales de acceso y/o } \\
\text { mejora entre los empleados como elementos } \\
\text { fundamentales en la gestión de recursos } \\
\text { humanos de cualquier empresa. }\end{array}$ & $\begin{array}{l}\text { Bustamente et al. (2019), MERCO (2018), Olmedo y } \\
\text { Martínez (2011), Martínez y Olmedo (2009) }\end{array}$ \\
\hline $\begin{array}{l}\text { Compromiso con } \\
\text { la comunidad }\end{array}$ & $\begin{array}{l}\text { El grado de compromiso de la cooperativa con } \\
\text { la comunidad vendrá determinado por el } \\
\text { número de actuaciones relacionadas con el } \\
\text { desarrollo de la comunidad donde actúa. }\end{array}$ & $\begin{array}{l}\text { MERCO (2018), Ponzi et al. (2011), Martín et al. } \\
\text { (2006), Helm (2005), Schwaiger (2004), Cravens et al. } \\
\text { (2003), De Quevedo (2003), Newell y Goldsmith } \\
\text { (2001) }\end{array}$ \\
\hline Credibilidad & $\begin{array}{l}\text { La credibilidad de la cooperativa resulta } \\
\text { imprescindible para crear confianza con los } \\
\text { grupos de interés y con el público en general, lo } \\
\text { cual es sinónimo de transparencia }\end{array}$ & $\begin{array}{l}\text { Bencomo y Fernández (2019), Iturrioz et al. (2019), } \\
\text { Maguregui et al. (2019), Schwaiger et al. (2019), } \\
\text { MERCO (2018), Olmedo y Martínez (2011), Helm } \\
\text { (2005, 2007), Schwaiger (2004), Fombrun et al. (2000), } \\
\text { Newell y Goldsmith (2001) }\end{array}$ \\
\hline $\begin{array}{l}\text { Proyección } \\
\text { internacional }\end{array}$ & $\begin{array}{l}\text { Operar a nivel internacional resulta un factor } \\
\text { clave para expandir la actividad de la } \\
\text { cooperativa y superar la frontera nacional. }\end{array}$ & $\begin{array}{l}\text { Colom et al. (2019), MERCO (2018), Villafañe (2004), } \\
\text { Olmedo y Martínez (2011), Villafañe }(2000,2004)\end{array}$ \\
\hline $\begin{array}{l}\text { Compromiso con } \\
\text { el sector }\end{array}$ & $\begin{array}{l}\text { La colaboración de la cooperativa en los } \\
\text { proyectos del sector es una cuestión } \\
\text { fundamental de cara a su legitimación en el } \\
\text { mismo. }\end{array}$ & $\begin{array}{l}\text { MERCO (2018), Cravens et al. (2003), Wessels (2003), } \\
\text { Newell y Goldsmith (2001) }\end{array}$ \\
\hline $\begin{array}{l}\text { Grado } \\
\text { identidad } \\
\text { cooperativa }\end{array}$ & $\begin{array}{l}\text { Por los principios cooperativos, resulta } \\
\text { fundamental el grado con el que los socios se } \\
\text { vinculan a la cooperativa. }\end{array}$ & $\begin{array}{l}\text { Cabaleiro et al. (2019), MERCO (2018), Olmedo y } \\
\text { Martínez (2011), Ponzi et al. (2011), Weigelt y } \\
\text { Camerer (1988) }\end{array}$ \\
\hline $\begin{array}{l}\text { Admiración } \\
\text { respeto }\end{array}$ & $\begin{array}{l}\text { La percepción de los grupos de interés externos } \\
\text { es de vital importancia para diferenciar a la } \\
\text { cooperativa en su entorno }\end{array}$ & $\begin{array}{l}\text { Colom et al. (2019), MERCO (2018), Olmedo y } \\
\text { Martínez (2011), Schwaiger (2004), Wessels (2003), } \\
\text { Fombrun et al. (2000) }\end{array}$ \\
\hline
\end{tabular}

Fuente: Elaboración propia

6 Concretamente, este ranking relaciona la relevancia de las cooperativas con su nivel de ventas y empleados, dejando fuera aspectos importantes a tratar en la valoración de la reputación https://www.faecta.coop/fileadmin/documentos/nacional/RANKING DE EMPRESAS MAS RELEVANTES DE LA ES 2016-2017 001.pdf (acceso octubre 2019).

Información disponible en: https://www.kornferry.com/haygroup (acceso noviembre 2019). 
Dentro de los indicadores seleccionados para la variable reputación mostrados en la Tabla 3, se incluyen aquellos que se relacionan tanto con la reputación financiera a través de resultados previstos, la reputación de productos a través de su calidad, la reputación empresarial estratégica y de gestión de recursos humanos y la reputación en responsabilidad social a través de la identidad, el respeto y la credibilidad.

\section{Metodología}

\subsection{Planteamiento de la investigación}

En esta investigación se ha hecho uso de un diseño metodológico por etapas. En la Figura 1 puede observarse cómo la primera etapa ha sido la revisión bibliográfica. En ella se han revisado las principales bases de datos SCOPUS y WOS así como las siguientes palabras clave: innovación, reputación, cooperativas, almazaras y aceite de oliva, todo ello para confeccionar un listado de indicadores para abordar la medición de la innovación y reputación (ya comentados en los apartados 2.3. y 3.1, respectivamente). Estos indicadores han sido la base para la subsiguiente aplicación de la técnica Delphi en una segunda etapa tendente a buscar su validación por los expertos en este ámbito.

Figura. 1. Modelo conceptual propuesto.

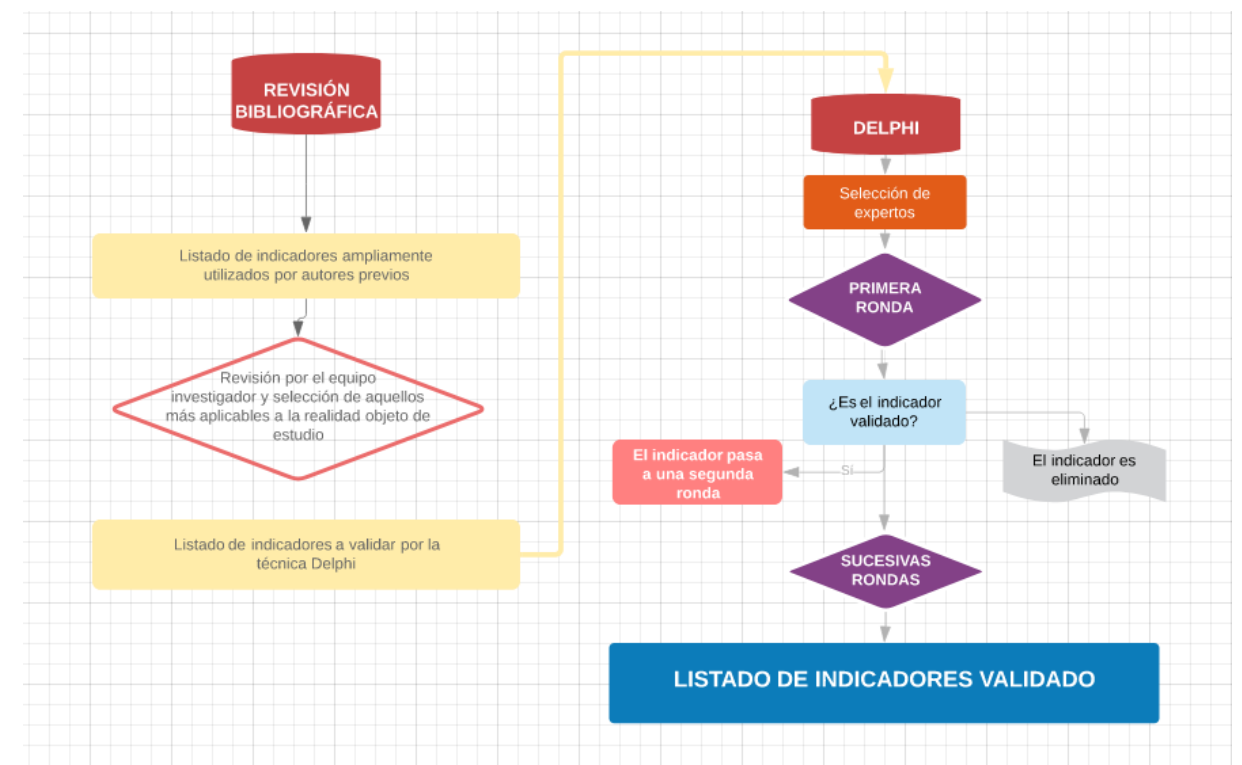

Fuente: Elaboración propia

\subsection{El uso de la Técnica Delphi}

La metodología Delphi se basa en la utilización sucesiva y sistemática del juicio consensuado de un grupo de expertos que remonta sus orígenes a los años 60 con el trabajo de Dalkey y Helmer (1963). Se trata de un método cualitativo donde el papel de las opiniones de los expertos en la materia se toma como base para llegar a una solución de consenso, generalmente relacionada con la previsión de acontecimientos futuros pero que también se ha empleado recientemente en una amplia variedad de problemáticas que incluyen la definición y validación de constructos de interés, elaborar instrumentos de análisis o recogida de información, entre otros (Cabero e Infante, 2014).

En nuestro caso, se elaboró un cuestionario inicial anónimo que incluía la descripción de todos los indicadores propuestos para las variables innovación (17 indicadores) y reputación (11 indicadores) tras la revisión bibliográfica realizada. A los expertos participantes se les planteó que valorasen la idoneidad o no de su utilización y justificando siempre su respuesta. Igualmente se añadió la posibilidad de los expertos añadiesen algunos indicadores que no estaban recogidos.

En total han participado 7 expertos procedentes del ámbito académico, del ámbito cooperativo y del sector del aceite de oliva, todos ellos con una sólida trayectoria y con gran reconocimiento y prestigio en sus respectivos campos. Si bien es cierto que la determinación de un número mínimo para la aplicación de esta 
técnica ha sido objeto de un intenso debate, nos situamos en el límite según Landeta (2002). En la Tabla 4 se recogen algunos datos descriptivos de los informantes seleccionados.

Tabla. 4. Perfil del experto participante

\begin{tabular}{|l|c|l|l|}
\hline Fases & Número de Participantes & \multicolumn{1}{c|}{ Sexo } & \multicolumn{1}{c|}{ Sector de actividad } \\
\hline Primera & 7 & Hombres: 4 & Académico: 2 \\
\cline { 1 - 2 } Segunda & 7 & Mujeres: 3 & Cooperativo y sectorial: 5 \\
\hline
\end{tabular}

Fuente: Elaboración propia

El lanzamiento del cuestionario inicial (primera ronda) fue a finales de 2018 por correo electrónico a cada uno de los expertos, si bien, su participación fue recabada mediante una conversación telefónica previa que aseguró que todos ellos formasen parte de, al menos, la primera ronda de contacto.

Tras el análisis de resultados se determinó necesaria la realización de una segunda ronda por parte del equipo investigador a principios de octubre de 2019. Se elaboró un segundo cuestionario anónimo donde se mostró a los participantes una modificación del listado inicial de indicadores que recogía las evidencias mayoritarias en torno a idoneidad o no de tales indicadores. Tras este segundo cuestionario los resultados obtenidos fueron refrendados y las variaciones encontradas resultaron mínimas por lo que no fue necesario continuar con una tercera vuelta dando por finalizada la aplicación de la técnica Delphi.

\section{Resultados}

Dedicamos este último bloque al comentario de los indicadores validados por la técnica Delphi para las variables innovación y reputación a las que dedicaremos un análisis individualizado.

\subsection{Indicadores válidos para la variable innovación cooperativa}

Los resultados obtenidos según rondas para cada uno de los indicadores propuestos aparecen detallados en la Tabla 5. En ella se han incorporado la puntuación obtenida y la permanencia o no del mismo. Debido a que los indicadores fueron planteados como variables dicotómicas, esto es, sí o no eran las dos únicas opciones de respuesta, la máxima puntuación posible (PMM en adelante) sería n (número de participantes de esa ronda)*1. De igual modo, la decisión de abandonar un indicador se justifica ante la no consecución de la puntuación media n (número de participantes de esa ronda)*1/2.

Tabla. 5. Indicadores para la variable innovación en las diferentes rondas del método Delphi ${ }^{8}$

\begin{tabular}{|c|c|c|c|c|c|}
\hline & INDICADOR PROPUESTO & $\begin{array}{l}\text { PRIMERA } \\
\text { RONDA } \\
\text { (PPM: } 7 \\
\text { PUNTOS) } \\
\end{array}$ & DECISIÓN & $\begin{array}{l}\text { SEGUNDA } \\
\text { RONDA } \\
\text { (PPM: } 7 \\
\text { PUNTOS) } \\
\end{array}$ & DECISIÓN \\
\hline \multirow{10}{*}{ 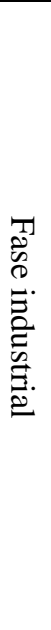 } & Incorporación de nuevos productos & 3 & Eliminar & & \\
\hline & $\begin{array}{l}\text { Modificación, mejora de productos } \\
\text { existentes }\end{array}$ & 6 & Mantener & 6 & Mantener \\
\hline & Incorporación de nuevos procesos & 6 & Mantener & \multirow[b]{2}{*}{7} & \multirow[b]{2}{*}{ Mantener } \\
\hline & $\begin{array}{l}\text { Modificación, mejora de procesos } \\
\text { productivos }\end{array}$ & 6 & \begin{tabular}{|c} 
Combinar con \\
hcorporación de nuevos \\
procesos
\end{tabular} & & \\
\hline & Certificaciones de calidad & 6 & Mantener & 7 & Mantener \\
\hline & Certificaciones ambientales & 6 & Mantener & 7 & Mantener \\
\hline & Certificaciones geográficas & 3 & Eliminar & & \\
\hline & Patentes & 6 & Mantener & 7 & Mantener \\
\hline & $\begin{array}{l}\text { Inversión en infraestructuras } \\
\text { innovadoras/tecnológicas }\end{array}$ & 7 & Mantener & 7 & Mantener \\
\hline & $\begin{array}{l}\text { Colaboraciones con universidad, } \\
\text { centros de investigación y otras }\end{array}$ & 7 & Mantener & 7 & Mantener \\
\hline
\end{tabular}

8 En rojo indicadores eliminados y en azul quedan los indicadores modificados. 


\begin{tabular}{|c|c|c|c|c|c|}
\hline & instituciones & & & & \\
\hline \multirow{7}{*}{ 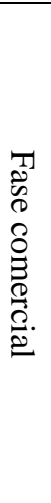 } & Sistemas de gestión de clientes & 7 & Mantener & 7 & Mantener \\
\hline & Número de marcas registradas & 5 & Mantener & \multirow[b]{2}{*}{6} & \multirow[b]{2}{*}{ Mantener } \\
\hline & Liderazgo en ventas envasadas & 5 & $\begin{array}{c}\text { Combinar con } \\
\text { "número de marcas } \\
\text { registradas" }\end{array}$ & & \\
\hline & Presencia ámbito comercio exterior & 6 & Mantener & 7 & Mantener \\
\hline & $\begin{array}{l}\text { Modificación sistema de } \\
\text { intercambio de información/TICs }\end{array}$ & 7 & Mantener & 7 & Mantener \\
\hline & $\begin{array}{l}\text { Responsabilidad Social asumida y } \\
\text { ejercida }\end{array}$ & 6 & Mantener & 7 & Mantener \\
\hline & $\begin{array}{l}\text { Visibilidad on line (número de } \\
\text { visitas) }\end{array}$ & 6 & Mantener & 7 & Mantener \\
\hline
\end{tabular}

Fuente: Elaboración propia

Al igual que en la tabla de indicadores, en las Figuras 2 y 3 también hemos distinguido la fase industrial y comercial de la variable innovación. De este modo, tal y como observamos en la Figura 2 a través de las sucesivas rondas de expertos el listado final propone eliminar los indicadores incorporación de nuevos productos y certificaciones geográficas, mientras que el indicador modificación y mejora de procesos productivos pasa a ser combinado con la incorporación de nuevos procesos.

Figura. 2. Indicadores de la variable innovación en la fase industrial: modificaciones Técnica Delphi.

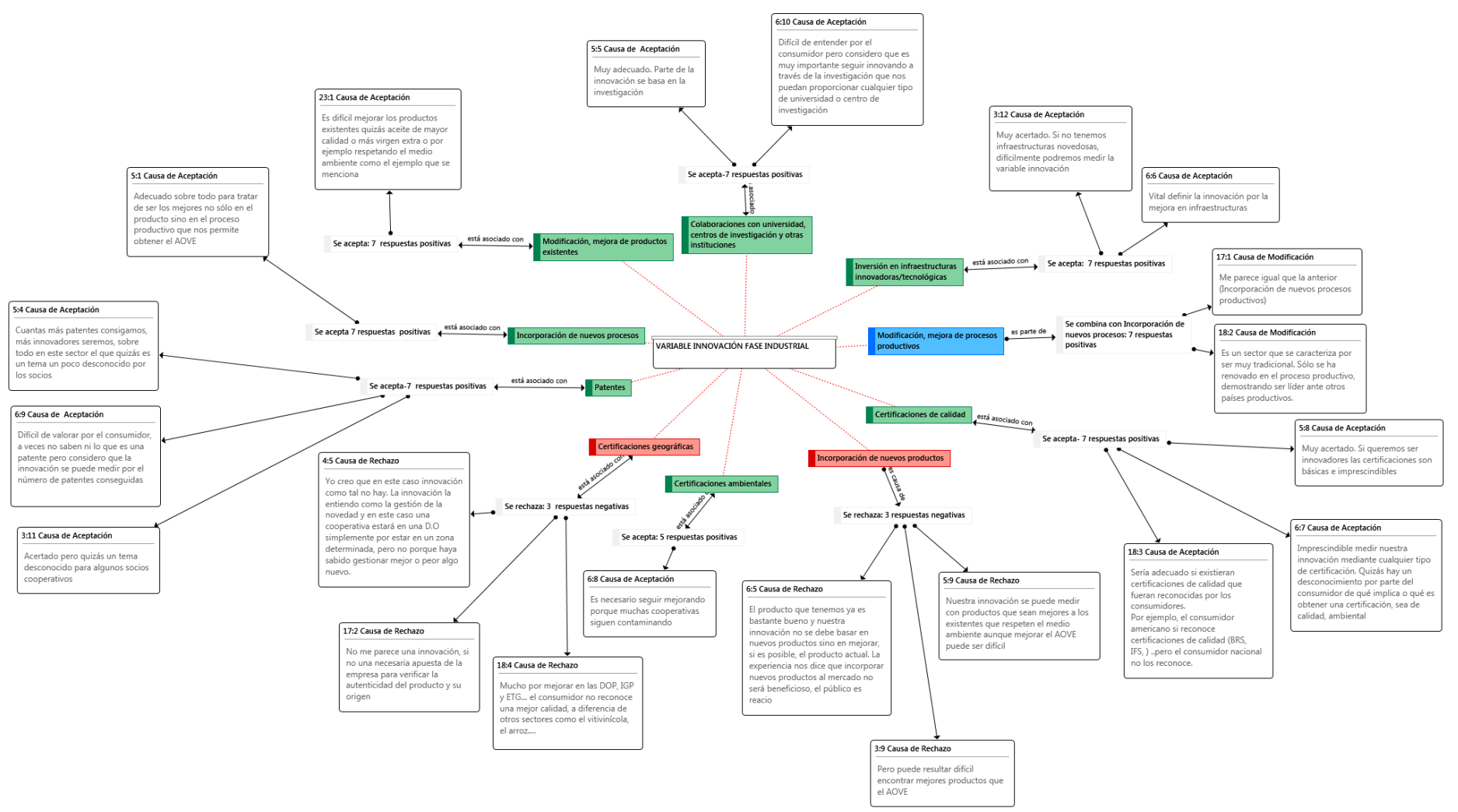

Fuente: Elaboración propia

Las opiniones que han justificado la eliminación del indicador "incorporación de nuevos productos" han resultado bastante homogéneas. Se cuestiona que la estrategia de las almazaras debe ser la mejora de sus aceites de oliva y no la de lanzar nuevos productos. El segundo indicador eliminado "Certificaciones geográficas" es considerado poco relacionado con la innovación al ser un atributo derivado del origen de la almazara, de nuevo las respuestas han sido muy consensuadas. El tercer cambio, la combinación de los indicadores "Incorporación de nuevos procesos productivos" y "Modificación, mejora de procesos 
productivos", se justifica porque conceptualmente los entrevistados entienden que son parecidos al resultar los primeros necesarios para modificar o mejorar los procesos productivos existentes.

La Figura 3 muestra los indicadores de la variable innovación en la fase comercial. En este sentido, apreciamos que no se propone la eliminación de ningún indicador, sino que la propuesta en base a la opinión de los expertos seleccionados consiste en combinar el indicador liderazgo en ventas envasadas con el número de marcas registradas.

Figura. 3. Indicadores de la variable innovación en la fase comercial: modificaciones Técnica Delphi

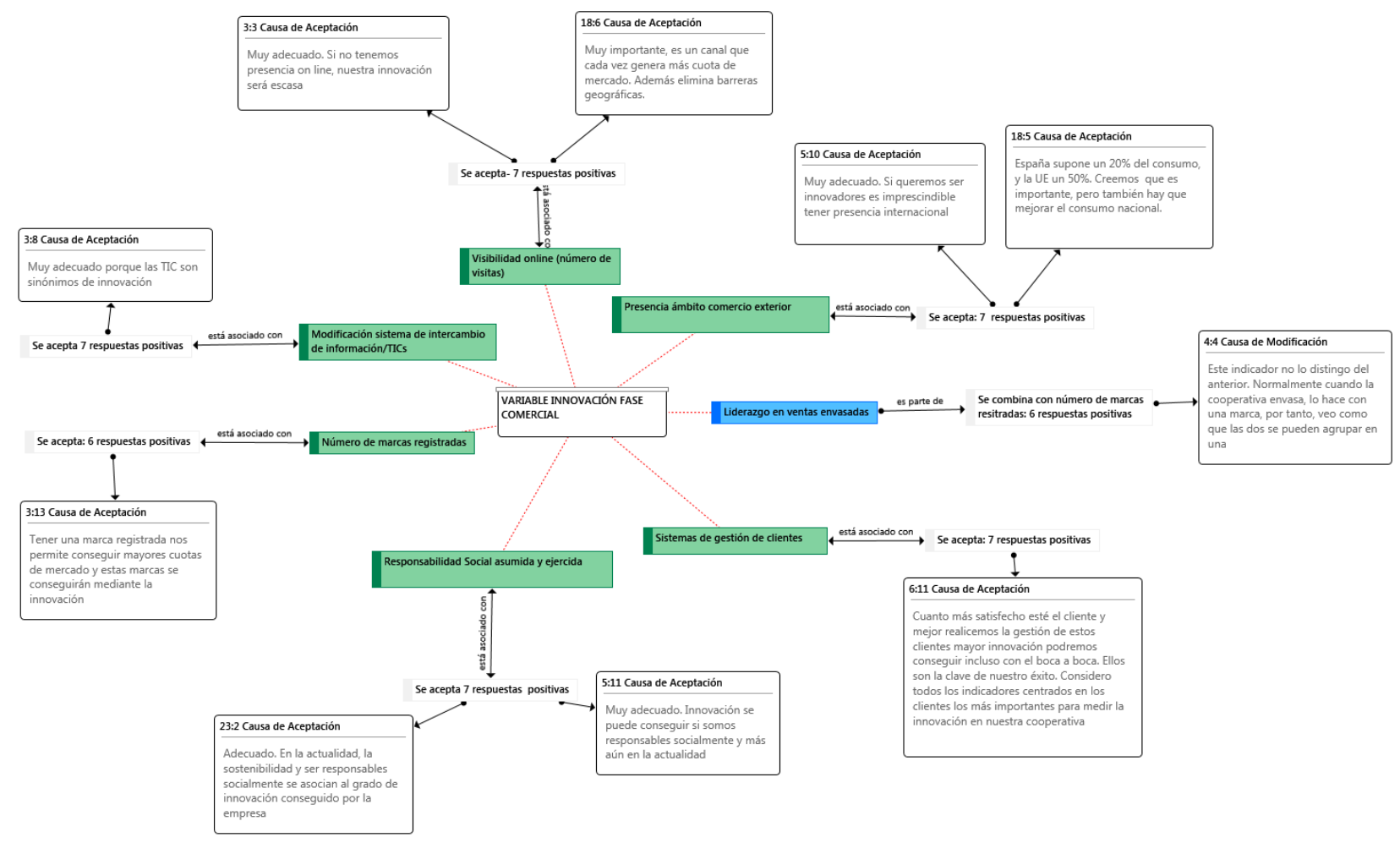

Fuente: Elaboración propia

La combinación de los indicadores "Número de marcas registradas" y "Liderazgo en ventas envasadas" ha quedado justificada por la consideración de los expertos sobre la inequívoca relación entre ambos, así cuando una almazara envasa lo hace a través de una marca registrada.

\subsection{Indicadores válidos para la variable reputación cooperativa}

Con relación a la variable reputación, los indicadores finalmente validados y sus puntuaciones obtenidas a lo largo de las sucesivas rondas pueden ser observados en la Tabla 6. Al igual que en el caso de la variable innovación, se solicitaba decidir sobre su adecuación o no a la realidad almazarera cooperativa, siendo la PMM calculada de forma análoga.

Tabla. 6. Indicadores para la variable reputación en las diferentes rondas del método Delphi ${ }^{9}$

\begin{tabular}{|l|c|c|c|c|}
\hline \multicolumn{1}{|c|}{ INDICADOR PROPUESTO } & $\begin{array}{c}\text { PRIMERA } \\
\text { RONDA (PPM: } \\
\text { 7 PUNTOS) }\end{array}$ & DECISIÓN & $\begin{array}{c}\text { SEGUNDA } \\
\text { RONDA } \\
\text { (PPM: } \\
\text { PUNTOS) }\end{array}$ & DECISIÓN \\
\hline $\begin{array}{l}\text { Proyección de beneficios a corto y } \\
\text { medio plazo }\end{array}$ & 5 & $\begin{array}{c}\text { Mantener modificando la } \\
\text { redacción en el sentido de } \\
\text { beneficios pasados }\end{array}$ & 7 & Mantener \\
\hline Calidad de los productos ofrecidos & 6 & Mantener & 7 & Mantener \\
\hline Acierto estrategia de la empresa & 6 & Mantener & 5 & Mantener \\
\hline
\end{tabular}

$9 \quad$ En azul los indicadores modificados. 


\begin{tabular}{|c|c|c|c|c|}
\hline Calidad laboral & 6 & Mantener & \multirow[b]{2}{*}{7} & \multirow{2}{*}{ Mantener } \\
\hline Igualdad de oportunidades & 5 & $\begin{array}{l}\text { - Combinar con "calidad } \\
\text { laboral" }\end{array}$ & & \\
\hline Compromiso con la comunidad & 7 & Mantener & 7 & Mantener \\
\hline Admiración y respeto & 7 & Mantener & \multirow[b]{2}{*}{7} & \multirow[b]{2}{*}{ Mantener } \\
\hline Credibilidad & 6 & $\begin{array}{c}\text { Combinar con "admiración y } \\
\text { respeto" }\end{array}$ & & \\
\hline Proyección internacional & 6 & Mantener & 7 & Mantener \\
\hline Compromiso con el sector & 6 & Mantener & 7 & Mantener \\
\hline Grado de identidad cooperativa & 6 & Mantener & 7 & Mantener \\
\hline
\end{tabular}

Fuente: Elaboración propia

Pasamos a comentar el listado validado finalmente para la variable reputación (véase Figura 4). Una vez analizados los indicadores por los expertos en la materia, éstos concluyen que no se debe eliminar ningún indicador, sino que proponen la combinación de algunos de ellos. En primer lugar, el indicador "Igualdad de oportunidades" y "Calidad laboral" se refieren a la misma temática, siendo el primero una señal de calidad en el trabajo. Por otro lado, "Credibilidad" y "Admiración y respeto" pueden abarcarse dentro de la confianza hacia la almazara, siendo el motivo que justificaría su reagrupación. En último lugar, se ha procedido a modificar el indicador "Proyección de beneficios a corto y medio plazo", al solicitar los expertos su reemplazo por una visión histórica.

Figura 4. Indicadores de la variable reputación: modificaciones Técnica Delphi.

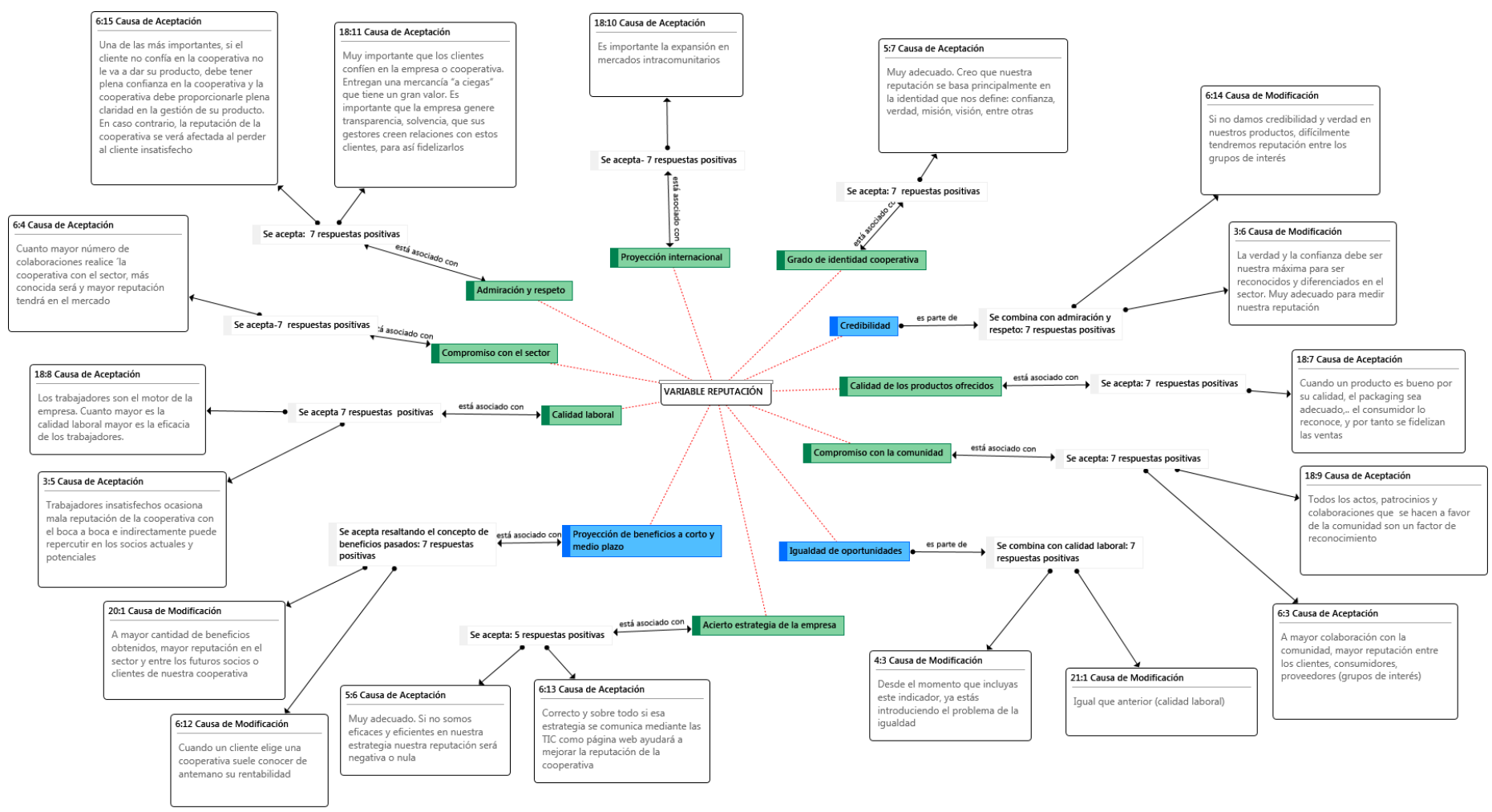

Fuente: Elaboración propia

\section{Conclusiones, limitaciones y futuras líneas de investigación}

La relación innovación-reputación ha constituido nuestro objetivo de investigación al entender que la relación que vincula a ambas variables es fundamental para el desarrollo competitivo y estratégico de la industria productora de aceite de oliva. Consideramos que la mejora de la reputación es fundamental para la diferenciación de estas empresas y, por este motivo, no sólo insistir en la misma sino promover su gestión a través del diseño de escalas para su medición resulta un importante avance. Con la propuesta de las escalas de medición realizada contribuimos a que los gerentes y directivos de almazaras cooperativas dispongan del 
conocimiento sobre cómo gestionar su innovación para que ésta pueda desencadenar en una mejor reputación de sus empresas. Igualmente, el ámbito académico tendrá una base para futuros estudios en la materia.

Como principales notas diferenciadoras de nuestro trabajo debemos indicar que se ha planteado la intangibilidad de ambas variables, innovación y reputación, a la vez que se ha justificado su consideración como constructos integrados por diferentes dimensiones y donde los indicadores no financieros han desempeñado un papel preponderante. De igual modo, se ha procedido a un diseño metodológico de dos fases: revisión bibliográfica y técnica Delphi, lo que garantiza que los indicadores propuestos y, finalmente validados, cuenten con el respaldo previo de autores en el campo. Finalmente, y como aportación clave, se han identificado un total de 13 indicadores que serían válidos para recoger la casuística de la innovación de almazaras cooperativas. De otro lado se han identificado 9 indicadores que nos permitirán acercarnos a la valoración de la reputación de estas empresas.

En cuanto a limitaciones, el modelo conceptual propuesto exige su contrastación empírica siendo precisamente lo que viene a determinar sus futuras líneas de investigación. Así, comprobar empíricamente la valoración de estas variables en una población de almazaras cooperativas representativa del tejido nacional, así como testar empíricamente el sentido positivo argumentado entre ambas variables, constituyen dos desarrollos del mismo que serán abordados en un futuro inmediato.

\section{Referencias bibliográficas}

Acevedo, N.M., Jiménez, L. M. y Rojas, M.D. (2016) Análisis bibliométrico sobre indicadores de innovación. Revista Espacios, Vol. 38, № 8, pp. 10-23.

Amo, M. (2018) La responsabilidad social empresarial como valor añadido en el sector oleícola. En: Hernández Ortiz, M.J. y Vilar Hernández, J. (2018) Algunas contribuciones de mejora en el ámbito de la dirección de empresas oleícolas, Fundación Caja Rural de Jaén, Jaén.

Anlló, G., Suárez, D. y De Angelis, J. (2009) Indicadores de innovación en América Latina: diez años del manual de Bogotá. Disponible en: http://www.ricyt.org/manuales/doc_view/83-indicadores-de-innovacion-en-america-latinadiez-anos-del-manual-de-bogota (Acceso octubre 2019).

Aponte, G.M. (2015) El proceso de gestión de innovación tecnológica: sus etapas e indicadores relacionados. Revista Venezolana de Análisis de Coyuntura, Vol. XXI, No 1, pp. 59-90.

Barney, J.B. (1991) Firm resources and sustained competitive advantage. Journal of Management, Vol. 17, N 1, pp. 99-120.

Bencomo, Y. y Fernández, D. (2019) Transparencia y rendición de cuentas: exigencias de la Responsabilidad Social de las Cooperativas no Agropecuarias en Cuba. REVESCO. Revista de Estudios Cooperativos, Segundo Cuatrimestre, $\mathrm{N}^{\circ}$ 131, pp. 179-198. https://doi.org/10.5209/REVE.62813.

Benito, S. y Esteban, P. (2012) La influencia de las políticas de responsabilidad social y la pertenencia a redes de cooperación en el capital relacional y estructural de las microempresas. Investigaciones Europeas de Dirección y Economía de la Empresa, Vol. 18, № 2, pp. 166-176.

Bergh, D.D., Ketchen, D.J., Boyd, B.K. y Bergh, J. (2010) New Frontiers of the Reputation-Performance Relationship: Insights from Multiple Theories. Journal of Management, Vol. 36, No 3, pp. 620-632.

Bernal, E., Mozas, A., Fernández, D., Medina, M.J. y Puentes, R. (2019) Calidad de los sitios web en el sector agroalimentario ecológico y sus factores explicativos: el papel del cooperativismo. CIRIEC-España, Revista de Economía Pública, Social y Cooperativa, No 95, pp. 95-118. https://doi.org/10.7203/CIRIEC-E.95.13207.

Bernal, E., Mozas, A., Medina, M.J. y Fernández, D. (2018) Evaluation of Corporate Websites and their influence on the performance of Olive Oil Companies. Sustainability, Vol. 10, No 4, pp. 1274-1285.

Bel, P. y Ausín, J.M. (2007) Contribución de las sociedades cooperativas al desarrollo territorial. REVESCO. Revista de Estudios Cooperativos, Segundo Cuatrimestre, $\mathrm{N}^{\circ}$ 92, pp. 41-71.

Boyd, B.A., Bergh, D.D. y Ketchen, D.J. (2010) Reconsidering the Reputation-Performance Relationship: A ResourceBased View. Journal of Management, Vol. 36, № 3, pp. 588-609.

Bretos, I., Díaz-Foncea, M. y Marcuelo, C. (2018) Cooperativas e internacionalización: un análisis de las 300 mayores cooperativas del mundo. CIRIEC-España, Revista de Economía Pública, Social y Cooperativa, No 92, pp. 5-37.

Bretos, I., Díaz-Foncea, M., Marcuello, C. y Marcuello, C. (2018b) Cooperativas, capital social y emprendimiento: Una perspectiva teórica. REVESCO. Revista de Estudios Cooperativos, Segundo Cuatrimestre, No 128, pp. 76-98. http://dx.doi.org/10.5209/REVE.59775.

Buenechea-Elberdin, M. (2017) Structured literature review about intellectual capital and innovation. Journal of Intellectual Capital, Vol. 28, $\mathrm{N}^{\circ}$ 2, pp. 262-285.

Bustamente, A.M. (2019) Gestión humana socialmente responsable en cooperativas de trabajo asociado colombianas. CIRIEC-España, Revista de Economía Pública, Social y Cooperativa, $\mathrm{N}^{\circ} \quad 95, \quad$ pp. 217-255. https://doi.org/10.7203/CIRIEC-E.95.10433.

Cabaleiro, M.J., Iglesias, C. y Martínez, R. (2019) Empresas democráticas y éxito económico. El modelo cooperativo. REVESCO. Revista de Estudios Cooperativos, Tercer Cuatrimestre, $\mathrm{N}^{\circ}$ 132, pp. 29-45. https://doi.org/10.5209/reve.65482. 
Caballer, V. y Moncaleano, G. (2004) Las nuevas tecnologías de la información en las cooperativas. Una aplicación a las cooperativas de crédito y citrícolas de la Comunidad Valenciana. CIRIEC-España, Revista de Economía Pública, Social y Cooperativa, $\mathrm{N}^{\circ}$ 49, pp. 239-261.

Cabero, J. e Infante, A. (2014) Empleo del método Delphi en la investigación en comunicación y educación. EDUTEC, Revista Electrónica de Investigación Educativa, № 48, pp. 1-16.

Cabrera, E.R., Arriaza, M. y Rodríguez-Entrena, M. (2015) Is the extra virgin olive oil market facing a process of differentiation?" Grasas y Aceites, Vol. 66, № 4, pp. 1-13.

Calabria, I. (2018) Diferenciación y singularización como estrategia competitiva para el olivar tradicional. En: Hernández Ortíz, M.J. y Vilar Hernández, J. (2018) Algunas contribuciones de mejora en el ámbito de la dirección de empresas oleícolas, Fundación Caja Rural de Jaén, Jaén.

Cao, Y., Myers, J.N., Myers, L.A. y Omer, T.C. (2015) Company reputation and cost of equity capital. Review of Accounting Studies, No 20, pp. 42-81.

Caravaca, I., González, G. y Mendoza, A. (2006) Indicadores de dinamismo, innovación y desarrollo. Su aplicación en ciudades pequeñas y medianas de Andalucía. Boletín de la A.G.E., No 43, pp. 131-154.

Carter, S. y Ruefli, T. (2006) Intra-Industry Reputation Dynamics under a Resource-Based Framework: Assessing the Durability Factor. Corporate Reputation Review, Vol. 9, $\mathrm{N}^{\mathrm{o}} \quad 1, \quad$ pp. 3-25. https://doi.org/10.1057/palgrave.crr.1550006.

Carrillo, I., Casado, J. y Pulido, J.I. (2019) Características de la oferta de oleoturismo. Estructuración interna para operar en el mercado turístico. Disponible en: http://www.revistaespacios.com/a19v40n32/19403225.html (Acceso octubre 2019).

Caruana, A. y Chircop, S. (2000) Measuring Corporate Reputation: A Case Example. Corporate Reputation Review, Vol. 3, No 1, pp. 43-57.

Casimiro, M.G. y Matos, A. (2015) El impacto de la reputación en el desempeño de la organización en la perspectiva de los miembros de las cooperativas. ESIC Market Economics and Business Journal, Vol. 46, No 1, pp. 37-65.

Castilla-Polo, F., Sánchez-Hernández, M.I., Gallardo-Vázquez, D. y Ruiz-Rodríguez, M.C. (2016) Diseño de un modelo de reputación para cooperativas. Revista de Contabilidad, Spanish Accounting Review, Vol. 19, № 1, pp. $154-167$. DOI: $10.1016 /$ j.rcsar.2015.10.003.

Castilla-Polo, F., Gallardo-Vázquez, D., Sánchez-Hernández, M.I. y Ruiz-Rodríguez, M.C. (2018) An empirical approach to analyze the reputation-performance linkage in agrifood cooperatives. Journal of Cleaner Production, $\mathrm{N}^{\circ}$ 195, pp. 163-175. https://doi.org/10.1016/j.jclepro.2018.05.210.

Castilla-Polo, F. y Ruiz-Rodríguez, M.C. (2018) Intangible Assets Disclosures in the Olive Oil Differentiation Strategy: A Theoretical Review. Agricultural Research and Technology, Vol. 14, $\mathrm{N}^{\mathrm{o}} \quad 1, \quad \mathrm{pp} . \quad 1-8$. 10.19080/ARTOAJ.2018.14.555904.

Chiavaro, E. (2014) A new decanter generation leads to innovation in olive oil Processing. European Journal of Lipid Science and Technology, № 116, pp. 1615-1617.

Chun, R. (2006) Innovation and Reputation: An Ethical Character Perspective. Creativity and Innovation Management, Vol. 15, No 1, pp. 63-73.

Colom, A., Cos, P. y Florensa, R.M. (2019) Cooperativismo agroalimentario en Europa. Dimensión, gobernanza y análisis BCG de las sociedades cooperativas TOP25 de la UE-28 y TOP10 en España. REVESCO. Revista de Estudios Cooperativos, Primer Cuatrimestre, No 130, pp. 73-98. http://dx.doi.org/10.5209/REVE.62811.

Corporate Excellence (2019) Approaching the Future $2019 . \quad$ Disponible en: https://content.gnoss.ws/doclinks/b3/b3ab/b3ab6273-9fdc-7647-e5f9-3780f124e4ea/es/tendencias-en-reputacion-ymarca--approaching-the e6e53b3a-9b06-cdd0-76f0-07775935a07d.pdf (Acceso noviembre 2019).

Cotec (2017) Innovación en España. Informe COTEC, Madrid. Disponible en: https://cotec.es/media/INFORMECOTEC-2017_versionweb.pdf (Acceso noviembre 2019).

Courtright, J. y Smudde, P.M. (2009) Leveraging Organizational Innovation for Strategic Reputation Management. Corporate Reputation Review, Vol. 12, № 3, pp. 245-269.

Cravens, K., Goad, E. y Ramamoorti, S. (2003) The Reputation Index: Measuring and Managing Corporate Reputation. European Management Journal, Vol. 21, N 2, pp. 201-212.

Dalkey, N. y Helmer, O. (1963) An experimental application of the Delphi method to the use of experts. Management Science, Vol. 9, No 3, pp. 458-467.

De Goey, H., Hilletofh, P. y Eriksson, L. (2019) Design-driven innovation: a systematic literature review. European Business Review, Vol. 31, № 1, pp. 92-114. https://doi.org/10.1108/EBR-09-2017-0160.

De Sá, J. (2012) Las prácticas de gestión de marketing en las cooperativas portuguesas. CIRIEC-España. Revista de Economía Pública, Social y Cooperativa, No 76, pp. 199-227.

De Quevedo, E. (2003) Reputación y creación de valor. Una relación circular. Madrid: Paraninfo.

De Toro, C. (2017) La innovación tecnológica en el sector oleícola español. En: Parras, M. y Gómez-Limón, M. (Coord.) (2017) Economía y comercialización de los aceites de oliva. Factores y perspectivas para el liderazgo español del mercado global. Fundación Cajamar, monografía № 31, pp. 413-427.

Dias, J. (2018) Resilient alliances: Portuguese cooperative movement in the transition to modern times (1867-1933). REVESCO. Revista de Estudios Cooperativos, Segundo Cuatrimestre, $\mathrm{N}^{\mathrm{o}} \quad 128, \quad$ pp. $59-75$. http://dx.doi.org/10.5209/REVE.60733. 
Dowling, G.R. (2004) Journalist evaluation of corporate reputation. Corporate Reputation Review, Vol. 7, № 2, pp. 196-205.

Escobar, A., Velandia, G. y Navarro, E. (2018) Gestión del conocimiento y competitividad en las cooperativas con sección de ahorro y crédito. REVESCO. Revista de Estudios Cooperativos, Primer Cuatrimestre, Vol. 127, pp. 90115. https://doi.org/10.5209/REVE.59769.

Escuela de Negocios Industrial (EOI) (2007) Identificación de criterios e indicadores de la excelencia en la gestión de la innovación en España. Disponible en: https://www.eoi.es/es/savia/publicaciones/19652/identificacion-decriterios-e-indicadores-de-la-excelencia-en-la-gestion-de-la-innovacion-en-espana. (Acceso noviembre 2019).

Etxezarreta, E., Mendiguren, J.C.P., Días, L. y Errasti, A. (2018) Valor social de las cooperativas sociales: aplicación del modelo poliédrico en la cooperativa para la acogida de menores Zabalduz S. Coop. CIRIEC-España, Revista de Economía Pública, Social y Cooperativa, No 93, pp. 155-180. https://doi.org/10.7203/CIRIEC-E.93.9953.

EXTENDA (2017) Estudio del sector del aceite de oliva en Andalucía. Disponible en: https://www.extenda.es/wpcontent/uploads/2018/01/ESTUDIO-DEL-ACEITE-DE-OLIVA-EN-ANDALUCIA-2017.pdf. (Acceso noviembre 2019).

Federación Andaluza de Empresas Cooperativas de Trabajo (FAECTA) (2016) Proyecto “La Innovación Social y las Cooperativas: el impacto social de las cooperativas y experiencias innovadoras socialmente”. Disponible en: https://www.faecta.coop/fileadmin/documentos/PDF_FAECTA/faecta_Informe_INNOVACION_SOCIAL_DEFINI TIVO.pdf (Acceso noviembre 2019).

Fajardo, G. (2018) La identificación de las empresas de economía social en España. Problemática jurídica. REVESCO. Revista de Estudios Cooperativos, Segundo Cuatrimestre, No 128, pp. 99-126. https://doi.org/10.5209/REVE.60209.

Fernández, A. y López, A. (2013) Validación mediante método Delphi de un sistema de indicadores para prever, diseñar y medir el impacto sobre el desarrollo local de proyectos de investigación en el sector agropecuario. Revista Ciencias Técnicas Agropecuarias, Vol. 22, № 3, pp. 54-60.

Fernández, D., Bernal, E., Mozas, A., Medina, M.J. y Moral, E. (2015) El sector cooperativo oleícola y el uso de las TIC: un estudio comparativo respecto a otras formas jurídicas. REVESCO. Revista de Estudios Cooperativos, Primer Cuatrimestre, № 120, pp. 53-75. https://doi.org/10.5209/rev_REVE.2016.v120.49700.

Fernández, D., Mozas, A., Bernal, E. y Medina, M.J. (2016) Uso y eficiencia de la social media. Un análisis desde la economía social. CIRIEC-ESPAÑA, Revista de Economía Pública, Social y Cooperativa, No , pp. 88: 5-27.

Fernández, M.V. y Peña, I. (2009) Estrategia de innovación como factor determinante del éxito de las cooperativas vitivinícolas de Castilla La Mancha. REVESCO. Revista de Estudios Cooperativos, Segundo Cuatrimestre, № 98, pp. 70-96.

Fidel, P., Schlesinger, W. y Espósito, E. (2018) Effects of customer knowlwdge management and customer orientation on innovation capacity and marketing results in $\mathrm{SME}^{\prime}$ : The mediating role of innovation orientation. International Journal of Innovation Management, Vol. 22, No 7. https://doi.org/10.1142/S136391961850055X.

Fombrun, C.J. (1996) Reputation: Realizing Value from Corporate Image. Boston, M.A.: Harvard Business School Press.

Fombrun, C.J., Gardberg, N.A. y Server, J.M. (2000) The reputation quotient sm: a multistakeholder measure of corporate reputation. The Journal of Brand Management, Vol. 7, № 4, pp. 241-255.

Fombrun, C.J. y Van Riel, C.B.M. (1998) The Reputational Landscape. Corporate Reputation Review, Vol. 1, № 1, pp. 5-14.

Fombrun, C.J. y Shanley, M. (1990) What's in a Name?: Reputation Building and Corporate Strategy. Academy of Management Journal, Vol. 33, No 2, pp. 233-258.

Foss, N.J. y Saebi, T. (2017) Fifteen Years of Research on Business Model Innovation: How Far Have We Come, and Where Should We Go?. Journal of Management, Vol. 43, $\mathrm{N}^{\mathrm{o}} \quad 1, \quad$ pp. $200-227$. http://dx.doi.org/10.1177/0149206316675927.

García, J.C., Iturrioz, J., Mateu, J.L. y Palomo, R. (2011) La percepción sobre la responsabilidad social en las sociedades cooperativas de trabajo asociado y las sociedades laborales: un análisis en el ámbito de la ciudad de Madrid. REVESCO. Revista de Estudios Cooperativos, Primer Cuatrimestre, No 104, pp. 102-124.

García, M., Alonso, A., Tello, M.L., Poza, M., Villalobos, N., Lansac, R. Mergarejo, P. y Laínez, M. (2018) Identifying agri-food research priorities for Spain -2017 results. Spanish Journal of Agricultural Research, Vol. 16, № 3, pp. 21-11.

Godino, F. (2018) Diseño de un sistema de gestión medioambiental para la industria productora de aceite de oliva. En: Hernández Ortiz, M.J. y Vilar Hernández, J. (2018) Algunas contribuciones de mejora en el ámbito de la dirección de empresas oleícolas. Fundación Caja Rural de Jaén, Jaén.

González, R. y Romeu, E. (2009) Medición de la innovación en el sector de las infocomunicaciones mediante indicadores basados en información de marcas. ACIMED, Vol. 19, $\mathrm{N}^{\mathrm{o}}$ 1. Disponible en: http://scielo.sld.cu/scielo.php?script=sci_arttext\&pid=S102494352009000100004\&lng=es\&nrm=iso ） (Acceso octubre 2019).

González-Ramos, M.I., Donate, M.J. y Guadamillas F. (2018) The effect of technological posture and Corporate Social Responsibility on financial performance thought corporate reputation. International Journal of Innovation, Vol. 6, $\mathrm{N}^{\mathrm{o}} 2$, pp. 164-179.

Grasenick, K. y Low, J. (2004) Shaken, not stirred. Defining and connecting indicators for the measurement and valuation of intangibles. Journal of Intellectual Capital, Vol. 5, № 2, pp. 268-281. 
Grimaldi, M., Corvello, V., De Mauro, A. y Scarmozzino, E. (2017) A systematic literature review on intangible assets and open innovation. Knowledge Management Research and Practice, Vol. 15, № 1, pp. 90-100.

Gutiérrez, H., y Palacios, P. D. (2015) Factores de la innovación y su influencia en las ventas y el empleo. El caso de las mipymes manufactureras mexicanas. Cuadernos de Economía, Vol. 34, № 65, pp. 401-422.

Hall, R.H. (1992) The Strategic Analysis of Intangible Resources. Strategic Management Journal, No 13, pp. $135-144$.

Hechevarria, Y. y Cruz, J. (2016) La innovación social cooperativa, una apuesta por construir una nueva economía social y solidaria. Revista de Cooperativismo y Desarrollo, Vol. 4, № 2, pp. 139-148.

Helm, S. (2005) Designing a Formative Measure for Corporate Reputation. Corporate Reputation Review, Vol. 8, № 2 , pp. 95-109.

Helm, S. (2007) One Reputation or Many? Comparing Stakeholders' Perceptions of Corporate Reputation. Corporate Communications: An International Journal, Vol. 12, No 3, pp. 238-254.

Henard, D.H. y Dacin, P.A. (2010) Reputation for Product Innovation: Its Impact on Consumers. Journal of Production Innovation Management, $\mathrm{N}^{\mathrm{o}} 27$, pp. 321-335.

Hölfinger, P.J., Nagel, C. y Sandner, P. (2018) Reputation for technological innovation: Does it actually cohere with innovative activity?. Journal of innovation and knowledge, Vol. $3, \quad \mathrm{~N}^{\mathrm{o}} \quad 1, \quad \mathrm{pp} . \quad 26-39$. https://doi.org/10.1016/j.jik.2017.08.002.

Iturrioz, J., Masa, C.I. y Medina, A. (2019) La transparencia sobre Responsabilidad Social Corporativa y su implicación con el comportamiento económico-financiero: efectos en las empresas de Economía Social. REVESCO. Revista de Estudios Cooperativos, Tercer Cuatrimestre, No 132, pp. 122-143. https://doi.org/10.5209/reve.65487.

Jafar, A. y Eshghi, T. (2011) The Role of Information and Communication Technology (ICT) in Iranian Olive Industrial Cluster. Journal of Agricultural Science, Vol. 3, No 1, pp. 228-232. https://doi.org/10.5539/jas.v3n1p228.

Jorge, J., Chivite, M. P. y Salinas, F. (2019) La transformación digital en el sector cooperativo agroalimentario español: situación y perspectivas. CIRIEC-España, Revista de Economía Pública, Social y Cooperativa, № 95, pp. 39-70. https://doi.org/10.7203/CIRIEC-E.95.13002.

Lajara-Camilleri, N. y Server-Izquierdo, R. (2017) ¿Cómo se puede mejorar la competitividad de las cooperativas agroalimentarias?. CIRIEC-España, Revista de Economía Pública, Social y Cooperativa, No 90, pp. $103-121$. https://doi.org/10.7203/CIRIEC-E.90.8854.

Landeta (2002): El método Delphi: una técnica de previsión para la incertidumbre. Barcelona: Ariel.

Lee, J. y Roh, J.J. (2012) Revisiting corporate reputation and firm performance link. Benchmarking: An International Journal, Vol. 19, № 4/5, pp. 649-664. https://doi.org/10.1108/14635771211258061.

López-Quesada, F.A. (2017) La Innovación en Marketing y Competitividad: Una aplicación al caso de las Cooperativas Agrícolas Españolas. Madrid: Esic Editorial.

López, V.A. e Iglesias, S. (2006) Percepciones directivas del recurso reputación. Estudio empírico de sus relaciones con el rendimiento empresarial. Cuadernos de Economía y Dirección de la Empresa, Vol. 28, pp. 139-160.

Lugones, G. (2009) Módulo de capacitación para la recolección y el análisis de indicadores de innovación. Banco Interamericano de Desarrollo. Disponible en: http://docs.politicascti.net/documents/Doc\%2008\%20\%20capacitacion\%20lugones\%20ES.pdf (Acceso octubre 2019).

Maestre Matos, L.M., Páez Cabas, A.P., Mesías, F.J. y Lombana Coy, J. (2019) Las cooperativas agrarias como modelo generador de negocios con inclusión social: el caso de las cooperativas bananeras del Magdalena (Colombia). REVESCO. Revista de Estudios Cooperativos, Tercer Cuatrimestre, N $^{\circ} \quad 132, \quad$ pp. $195-217$. http://dx.doi.org/10.5209/REVE.65488.

Maguregui, M.L., Corral, J. y Elechiguerra, C. (2019) La identificación de los grupos de interés de las entidades sin fines de lucro en la emisión de información transparente. REVESCO. Revista de Estudios Cooperativos, Segundo Cuatrimestre, Vol. 131, pp. 65-85. https://doi.org/10.5209/REVE.62814.

Marcuello, C. y Nachar, P. (2013) La sociedad cooperativa: motivación y coordinación. Un análisis desde las teorías económicas de la empresa y la economía social. REVESCO. Revista de Estudios Cooperativos, Primer Cuatrimestre, Vol. 110, pp. 192-222. https://doi.org/10.5209/rev_REVE.2013.v110.41440.

Marr, B., Schiuma, G. y Neely, A. (2004) The dynamics of value creation: mapping your intellectual performance drivers. Journal of Intellectual Capital, Vol. 5, No 2, pp. 312-325. https://doi.org/10.1108/14691930410533722.

Martín, G., Navas, J.E., López, P. (2006) Business and Social Reputation: Exploring the Concept and Main Dimensions of Corporate Reputation. Journal of Business Ethics, Vol. 63, № 4, pp. 361-370. https://doi.org/10.1007/s10551-0053244-Z.

Martínez-Carrasco, F. y Eid, M. (2017) El nivel de conocimiento y la reputación social de las empresas cooperativas. El caso de la Región de Murcia. CIRIEC-España, Revista de Economía Pública, Social y Cooperativa, No 91, pp. 5-29. https://doi.org/10.7203/CIRIEC-E.91.8865.

Martínez-García, I., Padillas-Carmona, M.T. y Suárez-Ortega, M. (2019) Aplicación de la metodología Delphi a la identificación de factores de éxito en el emprendimiento. Revista de Investigación Educativa, Vol. 37, № 1, pp. 129146. https://doi.org/10.6018/rie.37.1.320911.

Martínez, I.M. y Olmedo, I. (2009) La medición de la reputación empresarial: problemática y res-puesta. Investigaciones Europeas de Dirección y Economía de la Empresa, Vol. 15, No 2, pp. 127-142. https://doi.org/10.1016/S1135-2523(12)60093-8. 
Merco (2018) El proceso de elaboración de MERCO, 2018. Disponible en: file://C:/Users/Paqui/Downloads/metodologia-e-informe-de-verificacion-merco-empresas-es-2018\%20(1).pdf (Acceso noviembre 2019).

Meroño, A.L. y Arcas, N. (2006) Equipamiento y gestión de las tecnologías de la información en las cooperativas agroalimentarias. CIRIEC-España, Revista de Economía Pública, Social y Cooperativa, No 54, pp. 5-32.

Ministerio de Agricultura, Pesca y Alimentación (MAPA) (2004) Diagnóstico y análisis estratégico del sector agroalimentario español. Disponible en: https://www.mapa.es (Acceso octubre 2019).

Ministerio de Economía y Competitividad (2013) La Innovación en España según el Cuadro de Indicadores de la Unión por la Innovación de 2013. Disponible en: http://www.sodercan.es/wpcontent/uploads/sites/4/2015/06/INDICADORES-DE-LA-UNI\%C3\%93N-POR-LA-INNOVACION-2013.pdf (Acceso noviembre 2019).

Montegut, Y., Cristóbal, E. y Marimoni, F. (2007) Caracterización de las almazaras cooperativas catalanas. REVESCO. Revista de Estudios Cooperativos, Tercer Cuatrimestre, № 93, pp. 68-97.

Moradi, M. (2017) Investigating the Effect of Innovation on Effective Ways of Increasing Sales: Case Study of Dana Insurance. International Journal of Advanced Biotechnology and Research, Vol. 8, No 3, pp. 2092- 2099.

Moral, E., Bernal, E., Mozas, A. y Medina, M.J. (2014) Internacionalización del sector oleícola giennense. Mercados electrónicos. Instituto de Estudios Giennenses, Diputación de Jaén.

Mozas, A. y Puentes, R. (2010) La responsabilidad social corporativa y su paralelismo con las sociedades cooperativas. REVESCO. Revista de Estudios Cooperativos, Tercer Cuatrimestre, № 103, pp. 75-100.

Navarro, M., Gibaja, J.J., Franco, S., Murciego y A. Sáenz, J. (2011) Indicadores de innovación y benchmarking. Reflexión propuesta para el País Vasco. Bizkaia: Innobasque.

Newell, S.J., y Goldsmith, R.E. (2001) The development of a scale to measure perceived corporate credibility. Journal of Business research, Vol. 52, No 3, pp. 235-247. https://doi.org/10.1016/S0148-2963(99)00104-6.

Olmedo, I. y Martínez, I. (2011) Measure of corporate reputation in service SMEs. Revista Europea de Dirección y Economía de la Empresa, Vol. 20, N³, pp. 77-102.

Organization for Economic Cooperation and Development (OECD) (2005) Oslo Manual: Guidelines for Collecting and Interpreting Innovation Data, $3^{\circ}$ edición. The Measurement of Scientific and Technological Activities. Disponible en: http://dx.doi.org/10.1787/9789264013100-en.

Padgett, R.C. y Moura-Leitte, R.C. (2012) The impact of R\&D intensity on corporate reputation: Interaction effect of innovation with high social benefit. Intangible Capital, Vol. 8, No 2, pp. 216-238. http://dx.doi.org/10.3926/ic.336.

Parras, M. y Gómez-Limón, M. (Coord.) (2017) Economía y comercialización de los aceites de oliva. Factores y perspectivas para el liderazgo español del mercado global. Fundación Cajamar, monografía $\mathrm{N}^{\mathrm{o}} 31$ : http://www.publicacionescajamar.es/pdf/series-tematicas/informes-coyuntura-monografias/economia-ycomercializacion.pdf (Acceso octubre 2019).

Peraza-Castaneda, E.U., Gómez-García, J.-M. y Aleixandre-Mendizábal, G. (2016) Los factores determinantes del comportamiento innovador de las cooperativas: un análisis para el caso de Castilla y León. REVESCO. Revista de Estudios Cooperativos, Tercer Cuatrimestre, No 122, pp. 1-33. https://doi.org/10.5209/rev_REVE.2016.v122.52025.

Perea, E. (2009) Marcas y patentes como indicadores de innovación. Revista informador técnico, $\mathrm{N}^{\circ}$ 73, pp. 38-43. https://doi.org/10.23850/22565035.754.

Ponzi, L.J., Fombrun, C.J. y Gardberg, N.A. (2011) "RepTrak'TM Pulse: Conceptualizing and validating a short-form measure of corporate reputation. Corporate Reputation Review, Vol. 14, $\mathrm{N}^{\mathrm{o}}$ 1, pp. 15-35. https://doi.org/10.1057/crr.2011.5.

Porter, M. (1979) How competitive forces shape strategy. Harvard Business Review, Vol. 57, No 2, pp. 137-145.

Quesada, J. (2008) Política regional de innovación. Investigaciones regionales, $\mathrm{N}^{\mathrm{o}} \quad 12, \quad \mathrm{pp} . \quad 181-210$. https://doi.org/10.2775/74784.

Roberts, P.W. y Dowling, G.R. (2002) Corporate reputation and sustained superior financial performance. Strategic Management Journal, No 23, pp. 1077-1093. https://doi.org/10.1002/smj.274.

Ruiz, C. (2006) Disfunciones en el gobierno de las sociedades cooperativas agrarias: el caso de las almazaras cooperativas. GEZKI, No 2, pp. 73-103.

Sajardo-Moreno, A. y Chaves-Sajardo, R.J. (2017) Responsabilidad Social en las empresas de Economía Social: un análisis comparativo del tejido productivo de la Comunidad Valenciana. REVESCO. Revista de Estudios Cooperativos, Tercer Cuatrimestre, No 125, pp. 213-242. https://doi.org/10.5209/REVE.57065.

Sanchís, J.R. y Campos, V. (2008) La innovación social en la empresa. El caso de las cooperativas y de las empresas de Economía Social en España. Economía Industrial, № 368, pp. 187-196.

Sancho, R. (2002) Indicadores de los sistemas de ciencia, tecnología e innovación. Economía industrial, № 343, pp. 97 109.

Sancho, R. (2007) Innovación industrial. Revista Española de Documentación Científica, Vol. 30, № 4, pp. 553-564. https://doi.org/10.3989/redc.2007.v30.i4.405.

Sanz, J., Hervás, I., Sánchez, F. y Coq, D. (2011) Investigación e innovación en el sector del aceite de oliva en España. Disponible

http://www20.gencat.cat/docs/DAR/DE_Departament/DE02_Estadistiques_observatoris/24_Estudis_i_documents/0 1 Novetats documentals/Fitxers estatics/2012 NDW fitxers/NDW 120913 I+D+i_sector_oleicola_espanol.pdf (Acceso octubre 2019). 
Schwaiger, M. (2004) Components and parameters of corporate reputation-an empirical study. Schmalenbach Business Review, No 56, pp. 46-71. https://doi.org/10.1007/BF03396685.

Schwaiger, M., Manfred, J. y Enerhardt, S. M. (2019) How to Build a Corporate Reputation when You Don't Have One (Yet)? A Choice-Based Approach. 15 th EIASM Interdisciplinary conference on Intangibles and Intellectual Capital, Coimbra (Portugal).

Shafi'ei, Khankeh, H, Fallai, M. y Reza, P. (2017) Investigate the Effect of Acupressure on Fatigue Severity and Life Quality in Patients with Chronic Heart Failure. International Journal of Advanced Biotechnology and Research, Vol. 8, No 3, pp. 1649-1657. https://doi.org/10.1016/j.jams.2019.07.003.

Subramaniam, M. y Youndt, M.A. (2005) The influence of intellectual capital on the types of innovative capabilities. Academy of Management Journal, Vol. 48, No 3, pp. 450-463. https://doi.org/10.5465/AMJ.2005.17407911.

Valmohammadi, C. (2017) Customer relationship management: Innovation and performance. International Journal of Innovation Science, Vol. 9, № 4, pp. 374-395. https://doi.org/10.1108/IJIS-02-2017-001.

Vilar J., Velasco M.M. y Puentes, R. (2010) Incidencia del modo de explotación del olivo sobre la renta neta del olivicultor. Estrategias para el cultivo extensivo en el contexto de la posible ausencia de subvenciones. Grasas y Aceites, No 61, pp. 430-440. https://doi.org/10.3989/gya.010210

Vilar, J., Velasco, M.M., Puentes, R. y Martínez, A. (2011) El olivar tradicional: alternativas estratégicas de competitividad. Grasas y Aceites, Vol. 62, No 2, pp. 221-229. https://doi.org/10.3989/gya.091610. 221.

Villafañe, J. (2000) La reputación corporativa”, en J. Villafañe: El estado de la publicidad y el corporate en España. Madrid: Pirámide.

Villafañe, J. (2004) La buena reputación. Clave del valor intangible de las empresas. Madrid: Pirámide.

Walsh, G. y Beatty, S.E. (2007) Customer-Based Corporate Reputation of a Service Firm: Scale Development and Validation. Journal of Academy of Marketing Science, Vol. 35, № 1, pp. 127-143. https://doi.org/10.1007/s11747007-0015-7.

Wang, D., Yu, T. y Chiang, C. (2016) Exploring the value relevance of corporate reputation: A fuzzy-set qualitative comparative analysis. Journal of Business Research, Vol. 69, № 4, pp. $1329-1332$. https://doi.org/10.1016/j.jbusres.2015.10.101.

Wang, X. y Xu, M. (2018) Examining the linkage among open innovation, customer knowledge management and radical innovation. Baltic Journal of Management, Vol. 13, $\mathrm{N}^{\circ} 3$, pp. 368-389. https://doi.org/10.1108/BJM-042017-0108.

Weigelt, K. y Camerer, C. (1988) Reputation and Corporate Strategy: A Review of Recent Theory and Applications. Strategy Management Journal, Vol. 9, No 5, pp. 443-454. https://doi.org/10.1002/smj.4250090505.

Wessels, C. (2003) Reputation Defined. CSR Magazine, Vol. 2, No 3, pp. 28-29.

Zhang, X. (2018) Developing a New Measure of Media Reputation. Corporate Reputation Review, Vol. 21, № 2 , pp. 71-83. https://doi.org/10.1057/s41299-018-0043-X. 